\title{
Gravitational instability of finite isothermal spheres in general relativity. Analogy with neutron stars
}

\author{
P. H. Chavanis ${ }^{\star}$
}

\begin{abstract}
Laboratoire de Physique Quantique, Université Paul Sabatier, 118 route de Narbonne, 31062 Toulouse, France Institute for Theoretical Physics, University of California, Santa Barbara, California CA93106, USA
\end{abstract}

Received 29 August 2001 / Accepted 10 October 2001

\begin{abstract}
We investigate the effects of relativity on the gravitational instability of finite isothermal gaseous spheres. In the first part of the paper, we treat the gravitational field within the framework of Newtonian mechanics but we allow the speed of the particles to be close to the velocity of light so that special relativity must be taken into account. In the second part of the paper, we study the full general relativistic problem for a gas described by an equation of state $p=q \epsilon$ such that the pressure is proportional to the energy density ("isothermal" distribution). For $q=1 / 3$, this equation of state describes the core of neutron stars. The mass-density diagram displays some damped oscillations and there exists a critical value of mass-energy above which no equilibrium state is possible. We show analytically that the mass peaks are associated with new modes of instability. These results are strikingly similar to those obtained by Antonov (1962) and Lynden-Bell \& Wood (1968) for a classical isothermal gas. Our study completes the analogy between isothermal spheres and neutron stars investigated by Yabushita (1974).
\end{abstract}

Key words. hydrodynamics, instabilities - relativity - stars: neutron

\section{Introduction}

Isothermal spheres play an important role in astrophysics. They were initially introduced in the context of stellar structure (Chandrasekhar 1942) when composite configurations of stars consisting of an isothermal core and a polytropic envelope were constructed and studied. On larger scales, they were applied to stellar systems such as globular clusters and elliptical galaxies (Binney \& Tremaine 1987). The age of globular clusters is such that their isothermal structure is due to a succession of encounters between stars that lead to an equipartition of energy, like in an ordinary gas. This statistical mechanics prediction has been confirmed by direct observations of globular clusters and is well reproduced by King's models that incorporate a truncation in the distribution function so as to account for tidal effects. In the case of elliptical galaxies (and possibly other collisionless self-gravitating systems like massive neutrinos in Dark Matter models), the isothermal distribution is a result of a "violent relaxation" by phase mixing (Lynden-Bell 1967). In that case, there is no segregation by mass contrary to the collisional relaxation. In general, violent relaxation is incomplete and the distribution function must be modified at high energies. However, the distribution function that prevails in the inner regions of elliptical galaxies is isothermal and this is an

\footnotetext{
* e-mail: chavanis@irsamc2.ups-tlse.fr
}

important ingredient to understand de Vaucouleurs' $R^{1 / 4}$ law (Hjorth \& Madsen 1993). Isothermal distributions are also appropriate to describe the cold interstellar medium where the temperature is imposed by the cosmic background radiation at $T \sim 3 \mathrm{~K}$ in the outer parts of galaxies, devoid of any star and heating sources (Pfenniger \& Combes 1994; de Vega et al. 1996). They can also be of interest in cosmology to understand the fractal structure of the universe (Saslaw \& Hamilton 1984; de Vega et al. 1998).

The stability of isothermal gaseous spheres in Newtonian gravity has been investigated by Antonov (1962), Lynden-Bell \& Wood (1968) and Katz (1978) by using thermodynamical arguments and topological properties of the equilibrium phase diagram by analogy with Poincaré's theory of linear series of equilibrium. They showed in particular that, if the energy or the temperature are below a certain threshold, no hydrostatic equilibrium can exist. In that case, the system is expected to undergo a phase transition and collapse. This is called "gravothermal catastrophe" when the energy is kept fixed (microcanonical ensemble) and "isothermal collapse" when the system evolves at a fixed temperature (canonical ensemble). The thermodynamics of self-gravitating systems was reconsidered by Padmanabhan (1989) who calculated explicitly the second order variations of entropy and reduced the problem of stability to an eigenvalue equation. His method was applied in the canonical ensemble by Chavanis (2001) 
who showed in addition the equivalence between thermodynamical stability and dynamical stability based on the Navier-Stokes equations (Jeans problem). Similar studies have been performed by Semelin et al. (1999, 2000) and de Vega \& Sanchez (2001) by using field theoretical methods. As shown by Padmanabhan (1989) and Chavanis (2001), the problem of stability can be studied without approximation almost analytically (or with graphical constructions) by making use explicitly of the Milne variables introduced long ago in the context of stellar structure (Chandrasekhar 1942).

We show in the present paper that these methods naturally extend to the context of general relativity. Isothermal gaseous spheres (in the sense given below) have been only poorly studied in general relativity despite their similarity with classical isothermal spheres. The most extended study that we have found is the contribution of Chandrasekhar at the conference given in the honour of J. L. Synge in 1972. His work was completed by Yabushita $(1973,1974)$ who considered the stability of a relativistic isothermal gas surrounded by an external envelope imposing a constant pressure (an extension of the classical Bonnor 1956 problem). In order to make the link with the works of Antonov (1962) and Lynden-Bell \& Wood (1968) in Newtonian gravity, we shall consider the situation in which the volume is fixed instead of the pressure. This study can have direct applications to the stability of neutron stars since the equation of state that prevails in the central region of these highly relativistic objects is very close to the "isothermal" one.

In Sect. 2, we consider the stability of isothermal gas spheres in the framework of Newtonian gravity but taking into account special relativity. This is a first attempt to introduce relativistic effects in the classical Antonov problem. In fact, as is well-known, special relativity does not modify the equation of state for a perfect gas (Chandrasekhar 1942). Only does it alter the onset of instability. We find that the critical energy below which no hydrostatic equilibrium is possible depends on a relativistic parameter $\mu$ defined as the ratio between the size of the domain $R$ and a "classical" Schwarzschild radius $R_{\mathrm{S}}^{\text {class }}=2 G M / c^{2}$, where $M$ is the total mass of the system. In the classical limit $R \gg R_{\mathrm{S}}^{\text {class }}$, we recover the Antonov result $E_{\mathrm{c}}=-0.335 G M^{2} / R$ but when relativistic corrections are included we find that the critical energy is increased, i.e., instability occurs sooner than in the classical case. The density perturbation profile that triggers the instability is calculated explicitly. For $\mu \rightarrow+\infty$, it presents a "core-halo" structure but relativistic effects tend to reduce the extent of the halo. When the system is maintained at a fixed temperature instead of a fixed energy (canonical description), the results are unchanged with respect to the Newtonian case.

Of course, when the Schwarzschild radius becomes comparable to the size of the system, general relativistic effects must be taken into account. This problem is treated in detail in Sect. 3. We consider a simple equation of state $p=q \epsilon$ (where $q$ is a constant) which generalizes the equation of state for isothermal spheres in the Newtonian context (the classical limit is recovered for $q \rightarrow 0$ ). Since the equations governing equilibrium have the same structure and the same properties as in the classical case, we shall say that the system is "isothermal" (following the terminology of Chandrasekhar 1972), although this equation of state does not correspond to thermal equilibrium in a strict sense (see Sect. 3.2). True statistical equilibria in general relativity have been investigated by Katz et al. (1975) and they are characterized by a non uniform temperature (because of the gravitational redshift). However, systems described by the equation of state $p=q \epsilon$ are numerous in nature and they include for example the important case of neutron cores which are usually modeled as an assembly of cold degenerate fermions for which $q=1 / 3$. This simple isothermal equation of state is the high-density limit of more general equations of state usually considered for neutron stars (Oppenheimer \& Volkoff 1939; Meltzer \& Thorne 1966). Quite remarkably, our simple "box" model is able to reproduce the main properties of these objects. This suggests that the structure of neutron stars is due intrinsically to their isothermal cores and not to the details of their envelopes (which are not well-known). In Sect. 3.5, we show that the massdensity diagram displays an infinity of mass peaks and that isothermal spheres exist only below a limiting mass corresponding to the first peak. In Sect. 3.6, we show that the mass-radius diagram has a spiral behavior similar to the one observed for neutron stars. Using an equation of pulsation derived by Yabushita (1973), we demonstrate analytically that the series of equilibrium becomes unstable precisely at the point of maximum mass (like in the study of Misner \& Zapolsky 1964) and that new modes of instability correspond to secondary mass peaks. We obtain the same stability criterion from energy considerations based on the binding energy $E=M-N m c^{2}$. Said differently, these results indicate that, for a fixed mass, the system becomes unstable when its radius is smaller than a multiple of the Schwarzschild radius, a property consistent with Chandrasekhar's (1964) general theory. The perturbation profiles of density and velocity that trigger the instability are calculated explicitly and expressed in terms of the Milne variables. They do not present a "corehalo" structure. All these properties are strikingly similar to those obtained for classical isothermal spheres in the canonical ensemble (Chavanis 2001). This completes the analogy between isothermal spheres and neutron stars investigated by Yabushita (1974).

\section{Antonov instability for a relativistic gas sphere}

\subsection{The relativistic gas}

We consider a system of $N$ particles, each of mass $m$, in gravitational interaction. We allow the speed of the particles to be close to the velocity of light so that special relativity must be taken into account. However, in this first approach, we shall treat the gravitational field within 
the framework of Newtonian mechanics. This procedure is permissible if the typical size of the system is much larger than the Schwarzschild radius (see, e.g., Chandrasekhar 1942). Let $f(\boldsymbol{r}, \boldsymbol{p}, t)$ denote the distribution function of the system, i.e. $f(\boldsymbol{r}, \boldsymbol{p}, t) \mathrm{d}^{3} \boldsymbol{r} \mathrm{d}^{3} \boldsymbol{p}$ gives the average mass of particles whose positions and momenta are in the cell $\left(\boldsymbol{r}, \boldsymbol{p} ; \boldsymbol{r}+\mathrm{d}^{3} \boldsymbol{r}, \boldsymbol{p}+\mathrm{d}^{3} \boldsymbol{p}\right)$ at time $t$. The integral of $f$ over the momenta determines the spatial density

$\rho=\int f \mathrm{~d}^{3} \boldsymbol{p}$,

and the total mass is expressed as

$M=\int \rho \mathrm{d}^{3} \boldsymbol{r}$.

In a meanfield approximation, the total energy is given by

$E=\int \frac{f}{m} \epsilon \mathrm{d}^{3} \boldsymbol{r} \mathrm{d}^{3} \boldsymbol{p}+\frac{1}{2} \int \rho \Phi \mathrm{d}^{3} \boldsymbol{r}=K+W$,

where $K$ and $W$ are the kinetic and potential energy respectively. According to the theory of special relativity, the energy of a particule reads

$\epsilon=m c^{2}\left\{\left(1+\frac{p^{2}}{m^{2} c^{2}}\right)^{1 / 2}-1\right\}$.

This expression does not include the rest mass, so it reduces to the usual kinetic energy $\frac{p^{2}}{2 m}$ in the Newtonian limit. All the effects of gravity are incorporated in the potential energy which contains the gravitational potential $\Phi$ related to the density by the Newton-Poisson equation

$\Delta \Phi=4 \pi G \rho$.

We now ask which configuration maximizes the Boltzmann entropy

$S=-k \int \frac{f}{m} \ln \frac{f}{m} \mathrm{~d}^{3} \boldsymbol{r} \mathrm{d}^{3} \boldsymbol{p}$,

subject to the conservation of mass $M$ and energy $E$. To that purpose, we proceed in two steps. In this section, we maximize $S[f]$ at fixed $E, M$ and $\rho(\boldsymbol{r})$. This provides an optimal distribution function expressed in terms of $\rho$. Then, in Sect. 2.2, we maximize $S_{\max }[\rho]$ at fixed $M$ and $E$. This optimization problem is not trivial and will be further discussed in Sects. 2.4-2.6.

Since the gravitational potential can be deduced from the density by solving the Poisson Eq. (5), maximizing $S$ at fixed $E, M$ and $\rho(\boldsymbol{r})$ is equivalent to maximizing $S$ at fixed $\rho(\boldsymbol{r})$ and $K$. Writing the variational principle in the form

$\delta S-k \beta \delta K-\int \lambda(\boldsymbol{r}) \delta \rho \mathrm{d}^{3} \boldsymbol{r}=0$,

we obtain the optimal distribution function

$f(\boldsymbol{r}, \boldsymbol{p})=A(\boldsymbol{r}) \mathrm{e}^{-\beta \epsilon}$, which is a global entropy maximum with the previous constraints. Equation (8) is the relativistic MaxwellBoltzmann distribution with an inverse temperature

$\beta=\frac{1}{k T}$.

The Lagrange multipliers $A(\boldsymbol{r})$ and $\beta$ must be related to the constraints $\rho(\boldsymbol{r})$ and $K$. As discussed in detail by Chandrasekhar (1942), these relations can be expressed in terms of the modified Bessel functions

$K_{n}(z)=\int_{0}^{+\infty} \mathrm{e}^{-z \cosh \theta} \cosh (n \theta) \mathrm{d} \theta$.

Using Eqs. (1) (8) (4) and introducing the Juttner transformation $\frac{p}{m c}=\sinh \theta$ in the integral, the density can be written

$\rho(\boldsymbol{r})=\frac{4 \pi m^{3} c^{3}}{x} A(\boldsymbol{r}) K_{2}(x) \mathrm{e}^{x}$,

where we have introduced the dimensionless parameter

$x=\beta m c^{2}=\frac{m c^{2}}{k T}$.

This parameter quantifies the importance of relativistic effects. The classical limit corresponds to $x \rightarrow+\infty(k T \ll$ $\left.m c^{2}\right)$ and the ultra-relativistic limit to $x \rightarrow 0(k T \gg$ $m c^{2}$ ). The distribution function (8) can now be expressed in terms of the density as

$f(\boldsymbol{r}, \boldsymbol{p})=\frac{x}{4 \pi m^{3} c^{3}} \frac{\mathrm{e}^{-x}}{K_{2}(x)} \rho(\boldsymbol{r}) \mathrm{e}^{-\beta \epsilon}$.

In the classical limit $(x \rightarrow+\infty)$ we recover the standard formula

$f(\boldsymbol{r}, \boldsymbol{p})=\frac{1}{(2 \pi m k T)^{3 / 2}} \rho(\boldsymbol{r}) \mathrm{e}^{-\frac{p^{2}}{2 m k T}}$,

and in the ultra-relativistic limit $(x \rightarrow 0)$, we get

$f(\boldsymbol{r}, \boldsymbol{p})=\frac{c^{3}}{8 \pi k^{3} T^{3}} \rho(\boldsymbol{r}) \mathrm{e}^{-\frac{p c}{k T}}$.

Similarly, after some elementary transformations, the kinetic energy can be expressed in terms of the normalized inverse temperature $x$ by

$K=\mathcal{F}(x) M c^{2}, \quad \mathcal{F}(x)=\frac{3 K_{3}(x)+K_{1}(x)}{4 K_{2}(x)}-1$.

Using the recursion formula

$K_{n-1}(x)-K_{n+1}(x)=-\frac{2 n}{x} K_{n}(x)$,

the function $\mathcal{F}(x)$ can be written in the equivalent form

$\mathcal{F}(x)=\frac{K_{1}(x)}{K_{2}(x)}+\frac{3}{x}-1$.

It has the asymptotic behaviors

$\mathcal{F}(x) \sim \frac{3}{2 x} \quad(x \rightarrow+\infty)$, 
$\mathcal{F}(x) \sim \frac{3}{x} \quad(x \rightarrow 0)$.

When substituted in Eq. (16), we recover the usual expressions of the kinetic energy $K=\frac{3}{2} N k T$ in the classical limit and $K=3 N k T$ in the ultra-relativistic limit.

We can now express the entropy in terms of the density $\rho$ and the inverse temperature $x$. Substituting the optimal distribution function (13) in Eq. (6), we get, up to an additional constant

$S=k N \mathcal{G}(x)-k \int \frac{\rho}{m} \ln \frac{\rho}{m} \mathrm{~d}^{3} \boldsymbol{r}$,

where

$\mathcal{G}(x)=x[\mathcal{F}(x)+1]+\ln K_{2}(x)-\ln x$.

The function $\mathcal{G}(x)$ has the asymptotic behaviors

$\mathcal{G}(x) \sim-\frac{3}{2} \ln x \quad(x \rightarrow+\infty)$,

$\mathcal{G}(x) \sim-3 \ln x \quad(x \rightarrow 0)$.

The thermal contribution to the entropy in the classical limit is $S_{\mathrm{th}}=\frac{3}{2} k N \ln T$ and in the ultra-relativistic limit $S_{\mathrm{th}}=3 k N \ln T$.

There exists a general relation between the derivatives of $\mathcal{F}$ and $\mathcal{G}$ that we shall need in the following. Differentiating Eq. (22) with respect to $x$ and using the identity

$K_{n}^{\prime}(x)=-K_{n-1}(x)-\frac{n}{x} K_{n}(x)$,

for $n=2$, we find that

$\mathcal{G}^{\prime}(x)=x \mathcal{F}^{\prime}(x)$.

\subsection{First and second order variations of entropy}

In the preceding section, we have expressed the entropy and the kinetic energy in terms of the density $\rho(\boldsymbol{r})$ and the temperature $T$ (through the variable $x$ ). We now wish to maximize the entropy $S[\rho]$ at fixed $E$ and $M$. For convenience, we shall introduce a new variable $y=\mathcal{F}(x)$. In terms of this variable, the total energy and the entropy can be written

$E=y M c^{2}+\frac{1}{2} \int \rho \Phi \mathrm{d}^{3} \boldsymbol{r}$

$S=k N \mathcal{G}(x(y))-k \int \frac{\rho}{m} \ln \frac{\rho}{m} \mathrm{~d}^{3} \boldsymbol{r}$.

We can now determine the variations of $S$ around a given density profile $\rho(\boldsymbol{r})$. To second order in the expansion, we get

$$
\begin{aligned}
& \delta S=k N \frac{\mathrm{d} \mathcal{G}}{\mathrm{d} y} \delta y+k N \frac{\mathrm{d}^{2} \mathcal{G}}{\mathrm{d} y^{2}} \frac{(\delta y)^{2}}{2} \\
& -\frac{k}{m} \int \delta \rho\left(1+\ln \frac{\rho}{m}\right) \mathrm{d}^{3} \boldsymbol{r}-\frac{k}{m} \int \frac{(\delta \rho)^{2}}{2 \rho} \mathrm{d}^{3} \boldsymbol{r}
\end{aligned}
$$

Using the identity (26), we find that

$\frac{\mathrm{d} \mathcal{G}}{\mathrm{d} y}=\frac{\mathrm{d} \mathcal{G}}{\mathrm{d} x} \frac{\mathrm{d} x}{\mathrm{~d} y}=\frac{\mathcal{G}^{\prime}(x)}{\mathcal{F}^{\prime}(x)}=x$.

Differentiating one more time with respect to $y$, we obtain

$\frac{\mathrm{d}^{2} \mathcal{G}}{\mathrm{d} y^{2}}=\frac{\mathrm{d} x}{\mathrm{~d} y}=\frac{1}{\mathcal{F}^{\prime}(x)}$

Substituting the above results in Eq. (29), we get

$\delta S=k N x \delta y+k N \frac{1}{\mathcal{F}^{\prime}(x)} \frac{(\delta y)^{2}}{2}$

$-\frac{k}{m} \int\left(1+\ln \frac{\rho}{m}\right) \delta \rho \mathrm{d}^{3} \boldsymbol{r}-\frac{k}{m} \int \frac{(\delta \rho)^{2}}{2 \rho} \mathrm{d}^{3} \boldsymbol{r}$.

We now need to express the variation $\delta y$ in terms of $\delta \rho$. From the conservation of energy, we have the exact identity

$0=\delta E=M c^{2} \delta y+\int \Phi \delta \rho \mathrm{d}^{3} \boldsymbol{r}+\frac{1}{2} \int \delta \rho \delta \Phi \mathrm{d}^{3} \boldsymbol{r}$.

Substituting the foregoing expression for $\delta y$ from Eq. (33) in Eq. (32), we obtain

$$
\begin{aligned}
\delta S=- & \frac{1}{T} \int \Phi \delta \rho \mathrm{d}^{3} \boldsymbol{r}-\frac{1}{2 T} \int \delta \rho \delta \Phi \mathrm{d}^{3} \boldsymbol{r} \\
& +\frac{k}{2 M m c^{4}} \frac{1}{\mathcal{F}^{\prime}(x)}\left(\int \Phi \delta \rho \mathrm{d}^{3} \boldsymbol{r}\right)^{2} \\
& -\frac{k}{m} \int\left(1+\ln \frac{\rho}{m}\right) \delta \rho \mathrm{d}^{3} \boldsymbol{r}-\frac{k}{m} \int \frac{(\delta \rho)^{2}}{2 \rho} \mathrm{d}^{3} \boldsymbol{r} .
\end{aligned}
$$

Introducing a Lagrange multiplier $\alpha$ to satisfy the conservation of mass, the condition that $S$ is an extremum is written (to first order)

$0=\delta S-\alpha \delta M=-\int\left[\frac{\Phi}{T}+\frac{k}{m}\left(1+\ln \frac{\rho}{m}\right)+\alpha\right] \delta \rho \mathrm{d}^{3} \boldsymbol{r}$.

This condition must be satisfied for any variations $\delta \rho$. This yields the Boltzmann distribution

$\rho=A \mathrm{e}^{-\frac{m \Phi}{k T}}$

like for a classical gas. The condition that the critical point (36) is an entropy maximum requires that

$$
\begin{aligned}
\delta^{2} S & =-\frac{1}{2 T} \int \delta \rho \delta \Phi \mathrm{d}^{3} \boldsymbol{r}+\frac{k}{2 M m c^{4}} \\
& \times \frac{1}{\mathcal{F}^{\prime}(x)}\left(\int \Phi \delta \rho \mathrm{d}^{3} \boldsymbol{r}\right)^{2}-\frac{k}{m} \int \frac{(\delta \rho)^{2}}{2 \rho} \mathrm{d}^{3} \boldsymbol{r}<0,
\end{aligned}
$$

for any variation $\delta \rho$ that conserves mass to first order. 


\subsection{The Virial theorem for a relativistic gas}

In this section, we derive the form of the Virial theorem appropriate to an isothermal gas described in the framework of special relativity. Quite generally, the potential energy of a self-gravitating system can be expressed in the form (Binney \& Tremaine 1987)

$W=-\int \rho \boldsymbol{r} \nabla \Phi \mathrm{d}^{3} \boldsymbol{r}$.

If the system is in hydrostatic equilibrium, then

$\nabla p=-\rho \nabla \Phi$.

Substituting this identity in Eq. (38) and integrating by parts, we get

$W=\int \boldsymbol{r} \nabla p \mathrm{~d}^{3} \boldsymbol{r}=\oint p \boldsymbol{r} \mathrm{d}-3 \int p \mathrm{~d}^{3} \boldsymbol{r}$.

If the pressure $p_{\mathrm{b}}$ on the boundary of the system is uniform, we can write

$\oint p \boldsymbol{r} \mathrm{d}=p_{\mathrm{b}} \oint \boldsymbol{r} \mathrm{d} \boldsymbol{S}=p_{\mathrm{b}} \int \nabla \cdot \boldsymbol{r} \mathrm{d}^{3} \boldsymbol{r}=3 p_{\mathrm{b}} V$,

where $V$ is the total volume of the system. Therefore, for any system in hydrostatic equilibrium, one has

$W=3 p_{\mathrm{b}} V-3 \int p \mathrm{~d}^{3} \boldsymbol{r}$,

which can be considered as the general form of the Virial theorem for self-gravitating systems.

Now, the pressure of an ideal gas can be expressed as (Chandrasekhar 1942)

$p=\frac{1}{3} \int \frac{f}{m} p \frac{\partial \epsilon}{\partial p} \mathrm{~d}^{3} \boldsymbol{p}$.

For a relativistic gas described by the distribution function (8), we get

$p=-\frac{4 \pi}{3 m \beta} A(\boldsymbol{r}) \int_{0}^{+\infty} \frac{\partial}{\partial p}\left(\mathrm{e}^{-\beta \epsilon}\right) p^{3} \mathrm{~d} p$.

Integrating by parts, we obtain

$p=\frac{\rho}{m \beta}=\frac{\rho}{m} k T$.

Therefore, the equation of state for a (non quantum) relativistic gas is the same as for a classical gas. Written in the form

$W+3 N k T=3 p_{\mathrm{b}} V$,

the Virial theorem (42) also has the same form as for an isothermal classical gas. However, the Virial theorem is usually expressed in terms of the kinetic energy instead of the temperature. Therefore, the appropriate form of the relativistic Virial theorem reads

$W+\frac{3 M c^{2}}{\mathcal{F}^{-1}\left(\frac{K}{M c^{2}}\right)}=3 p_{\mathrm{b}} V$.
In the classical limit $(x \rightarrow+\infty)$, it reduces to the wellknown formula

$W+2 K=3 p_{\mathrm{b}} V$

and in the ultra-relativistic limit $(x \rightarrow 0)$, we get

$W+K=3 p_{\mathrm{b}} V$

It should be emphasized that Eq. (47) is only valid for a relativistic gas in thermal equilibrium.

\subsection{The equilibrium phase diagram}

Since the equation of state for a relativistic gas is the same as for a classical gas, the equilibrium configurations of such systems correspond to the isothermal gas spheres extensively described in the monograph of Chandrasekhar (1942). Only the onset of the gravitational instability will be modified by relativistic effects.

For non-rotating systems, the equilibrium states are expected to be spherically symmetric. In that case, the Poisson Eq. (5) together with the Boltzmann distribution (36) yield the second order differential equation

$\frac{1}{r^{2}} \frac{\mathrm{d}}{\mathrm{d} r}\left(r^{2} \frac{\mathrm{d} \Phi}{\mathrm{d} r}\right)=4 \pi G A \mathrm{e}^{-\beta m \Phi}$.

This equation can also be deduced from the condition of hydrostatic equilibrium (39) when the pressure is related to the density according to the equation of state (45). It is well-known that the density profile of such isothermal configuations behaves like $\rho \sim r^{-2}$ at large distances so that their total mass is infinite. Following Antonov (1962), we shall avoid this infinite mass problem by confining artificially the system within a spherical box of radius $R$. It is only under this simplifying assumption that a rigorous thermodynamics of self-gravitating systems can be carried out (see, e.g., Padmanabhan 1990; Chavanis et al. 2001). This procedure is justified physically by the realization that a distribution of matter never extends to infinity so $R$ represents an upper cut-off at which other processes intervene to limitate the spatial extent of the system. Of course, different cut-offs are possible but fixing the volume is consistent with the traditional viewpoint of statistical mechanics and it is sufficient to capture the essential physics of the problem (see the different comparisons of truncated models performed by Katz 1980).

We now wish to determine the equilibrium phase diagram of a relativistic isothermal gas. To that purpose, we introduce the function $\psi=\beta m\left(\Phi-\Phi_{0}\right)$ where $\Phi_{0}$ is the gravitational potential at $r=0$. Then, the density field (36) can be written

$\rho=\rho_{0} \mathrm{e}^{-\psi}$,

where $\rho_{0}$ is the central density. Introducing the notation $\xi=\left(4 \pi G \beta m \rho_{0}\right)^{1 / 2} r$, the Boltzmann-Poisson Eq. (50) reduces to the standard Emden form

$\frac{1}{\xi^{2}} \frac{\mathrm{d}}{\mathrm{d} \xi}\left(\xi^{2} \frac{\mathrm{d} \psi}{\mathrm{d} \xi}\right)=\mathrm{e}^{-\psi}$. 
Equation (52) has a simple analytic solution, the singular sphere

$\mathrm{e}^{-\psi_{\mathrm{s}}}=\frac{2}{\xi^{2}}$

whose central density is infinite. The regular solution of Eq. (52) satisfying the boundary conditions

$\psi(0)=\psi^{\prime}(0)=0$,

at the center of the sphere must be computed numerically. In the case of bounded isothermal systems, we must stop the integration at the normalized box radius

$\alpha=\left(4 \pi G \beta m \rho_{0}\right)^{1 / 2} R$.

We shall now relate the parameter $\alpha$ to the temperature and the energy. According to the Poisson Eq. (5), we have

$$
\begin{aligned}
& G M=\int_{0}^{R} 4 \pi G \rho r^{2} \mathrm{~d} r= \\
& \int_{0}^{R} \frac{\mathrm{d}}{\mathrm{d} r}\left(r^{2} \frac{\mathrm{d} \Phi}{\mathrm{d} r}\right) \mathrm{d} r=\left(r^{2} \frac{\mathrm{d} \Phi}{\mathrm{d} r}\right)_{r=R},
\end{aligned}
$$

which is just a particular case of the Gauss theorem. Introducing the dimensionless variables defined previously, we get

$\eta \equiv \frac{\beta G M m}{R}=\alpha \psi^{\prime}(\alpha)$.

The relation between $\alpha$ and the normalized temperature $\eta$ is not affected by special relativity.

For the total energy, using the Virial theorem (46) and the expression (16) for the kinetic term, we have

$E=K+W=\mathcal{F}(x) M c^{2}-\frac{3 N}{\beta}+3 p(R) V$.

Now, the pressure at the boundary of the domain can be written

$p(R)=\frac{\rho(R)}{m \beta}=\frac{\rho_{0} \mathrm{e}^{-\psi(\alpha)}}{m \beta}$.

Expressing the central density in terms of $\alpha$, using Eq. (55), we have equivalently

$p(R)=\frac{\alpha^{2}}{4 \pi G \beta^{2} m^{2} R^{2}} \mathrm{e}^{-\psi(\alpha)}$.

The total energy therefore reads

$\Lambda \equiv-\frac{E R}{G M^{2}}=-\frac{R c^{2}}{G M} \mathcal{F}(x)+\frac{3}{\alpha \psi^{\prime}(\alpha)}-\frac{\mathrm{e}^{-\psi(\alpha)}}{\psi^{\prime}(\alpha)^{2}}$,

where we have used Eq. (57) to eliminate the temperature in the last two terms.

It will be convenient in the following to introduce the parameter

$\mu=\frac{R c^{2}}{G M} \equiv \frac{2 R}{R_{\mathrm{S}}^{\text {class }}}$,

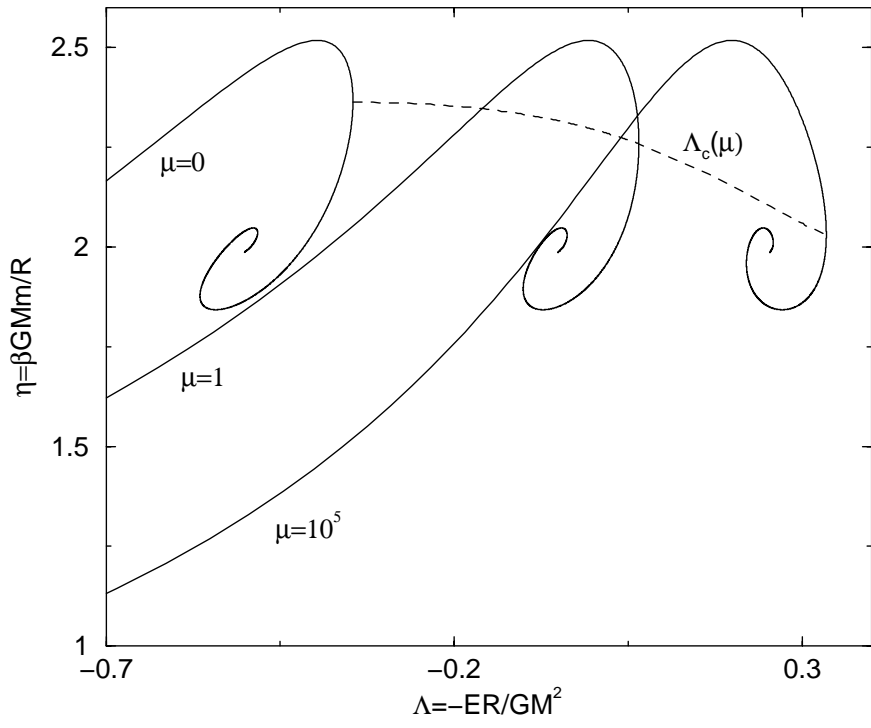

Fig. 1. Equilibrium phase diagram for isothermal gas spheres described in the framework of special relativity. Relativistic effects shift the onset of instability to larger energies.

which is twice the ratio between the system radius $R$ and the "classical" Schwarzschild radius

$R_{\mathrm{S}}^{\text {class }}=\frac{2 G M}{c^{2}}$,

constructed with the total mass $M$ of the system. Clearly, our semi-relativistic treatment, which ignores general relativity, is only valid for $\mu \gg 1$. However, in our rather formal analysis, we shall treat $\mu$ as a free parameter varying in the range $0 \leq \mu<+\infty$. The relativistic parameters $\mu$ and $x$ are related to each other by

$x=\mu \eta$.

Therefore, in accordance with Eqs. (61) and (64), the relation between the parameter $\alpha$ and the normalized energy $\Lambda$ takes the form

$\Lambda \equiv-\frac{E R}{G M^{2}}=-\mu \mathcal{F}\left[\mu \alpha \psi^{\prime}(\alpha)\right]+\frac{3}{\alpha \psi^{\prime}(\alpha)}-\frac{\mathrm{e}^{-\psi(\alpha)}}{\psi^{\prime}(\alpha)^{2}}$.

In the classical limit $(\mu \rightarrow+\infty)$, we recover the result of Lynden-Bell \& Wood (1968)

$\Lambda=\frac{3}{2 \alpha \psi^{\prime}(\alpha)}-\frac{\mathrm{e}^{-\psi(\alpha)}}{\psi^{\prime}(\alpha)^{2}}$,

and in the formal limit $\mu \rightarrow 0$, we get

$\Lambda=-\frac{\mathrm{e}^{-\psi(\alpha)}}{\psi^{\prime}(\alpha)^{2}}$.

In Fig. 1, we have represented the equilibrium phase diagram $\beta-E$ for different values of the relativistic parameter $\mu$. We see that the effect of relativity is to shift the spiral to the left. Therefore, the "gravothermal catastrophe" (corresponding to the absence of equilibrium below a critical energy) occurs sooner than in the Newtonian case. 


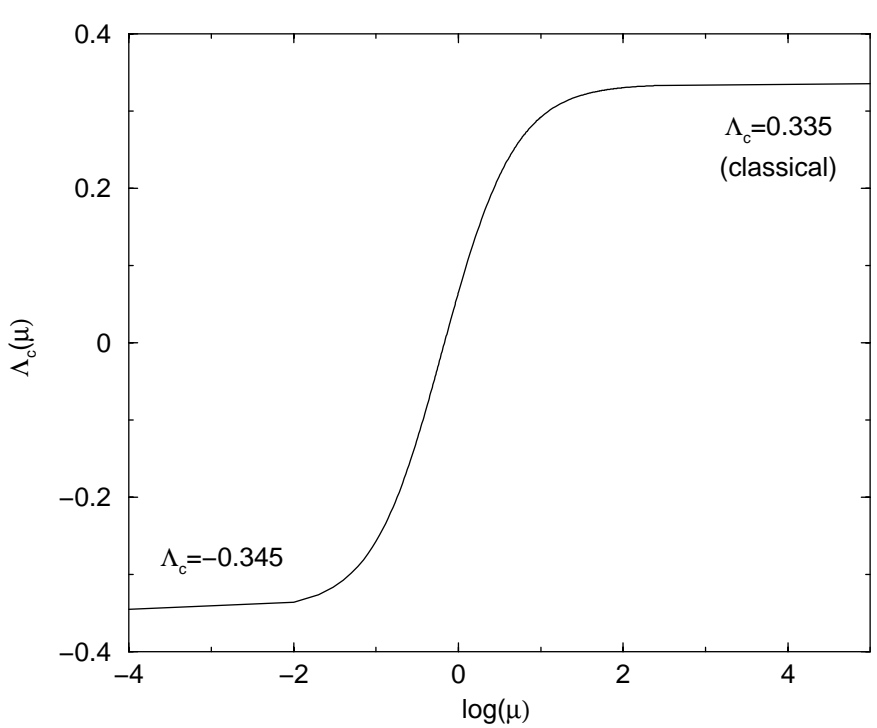

Fig. 2. Critical energy $\Lambda_{\mathrm{c}}$ as a function of the relativistic parameter $\mu$.

The critical energy is ploted as a function of the relativistic parameter $\mu$ in Fig. 2. For $\mu \rightarrow+\infty$, we recover the classical result of Antonov $\Lambda_{\mathrm{c}}(+\infty)=0.335$ and, in the formal limit $\mu \rightarrow 0$, we get $\Lambda_{\mathrm{c}}(0)=-0.345$. Clearly, the spiral is not destroyed by relativistic effects. Only is its shape slightly modified: the relativistic spirals are more "stretched" than the classical one. Note that the maximum value of $\eta$ is independant on $\mu$ and is equal to its classical value $\eta_{\mathrm{c}}=2.52$. On the other hand, substituting the expression (53) for the singular sphere in Eqs. (57) and (65), we find that the center of the spiral is determined by the equations

$\eta_{\mathrm{s}}=2$,

$\Lambda_{\mathrm{s}}(\mu)=1-\mu \mathcal{F}(2 \mu)$

In the limit $\mu \rightarrow+\infty, \Lambda_{\mathrm{s}}(+\infty)=\frac{1}{4}$ and in the limit $\mu \rightarrow 0, \Lambda_{\mathrm{s}}(0)=-\frac{1}{2}$.

The spiral is parametrized by the normalized box radius $\alpha$ that goes from 0 (ordinary gas) to $+\infty$ (singular sphere) when we spiral inwards. If one prefers, we can use a parametrization in terms of the density contrast

$\mathcal{R}=\frac{\rho_{0}}{\rho(R)}=\mathrm{e}^{\psi(\alpha)}$,

that goes from 1 to $+\infty$. In Fig. 3, we plot the critical density contrast (corresponding to $\Lambda_{\mathrm{c}}$ ) as a function of the relativistic parameter $\mu$. For $\mu \rightarrow+\infty$, we recover the classical value $\mathcal{R}_{\mathrm{c}}(+\infty)=709$ (and $\alpha_{\mathrm{c}}(+\infty)=34.4$ ). For $\mu=0$, we get $\mathcal{R}_{\mathrm{c}}(0)=132$ (and $\left.\alpha_{\mathrm{c}}(0)=16.0\right)$. It is found that instability occurs for smaller density contrasts when relativity is accounted for.

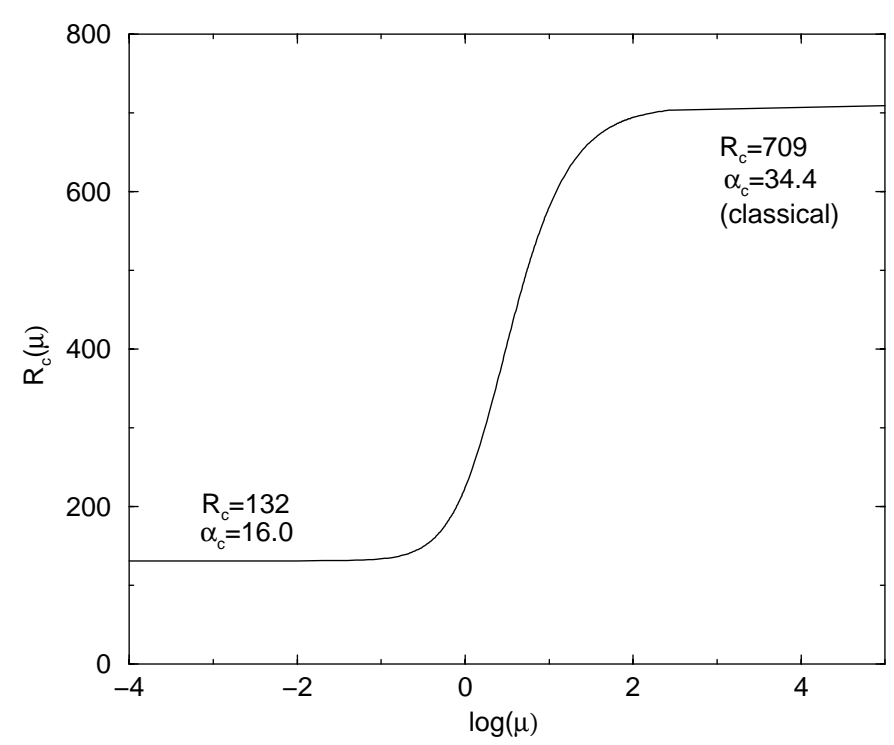

Fig. 3. Critical density contrast $\mathcal{R}_{\mathrm{c}}$ as a function of the relativistic parameter $\mu$.

\subsection{The Milne variables}

It will be convenient in the following to introduce the Milne variables $(u, v)$ defined by (Chandrasekhar 1942)

$u=\frac{\xi \mathrm{e}^{-\psi}}{\psi^{\prime}}, \quad$ and $\quad v=\xi \psi^{\prime}$.

Taking the logarithmic derivative of $u$ and $v$ with respect to $\xi$ and using Eq. (52), we get

$\frac{1}{u} \frac{\mathrm{d} u}{\mathrm{~d} \xi}=\frac{1}{\xi}(3-v-u)$

$\frac{1}{v} \frac{\mathrm{d} v}{\mathrm{~d} \xi}=\frac{1}{\xi}(u-1)$.

Taking the ratio of these equations, we find that the variables $u$ and $v$ are related to each other by a first order differential equation

$\frac{u}{v} \frac{\mathrm{d} v}{\mathrm{~d} u}=-\frac{u-1}{u+v-3}$.

The solution curve in the $(u, v)$ plane is well-known and is represented in Fig. 4. Its striking oscillating behavior has been described by a number of authors (see in particular Chandrasekhar 1942). We refer to Padmanabhan (1989) and Chavanis (2001) for the description of its main characteristics in connexion with the present work.

It turns out that the normalized temperature and the normalized energy can be expressed very simply in terms of the values of $u$ and $v$ at the normalized box radius $\alpha$. Indeed, writing $u_{0}=u(\alpha)$ and $v_{0}=v(\alpha)$ and using Eqs. (57), (65), we get

$\eta=v_{0}$

$\Lambda=-\mu \mathcal{F}\left(\mu v_{0}\right)+\frac{3}{v_{0}}-\frac{u_{0}}{v_{0}}$. 
The intersections between the curves defined by Eqs. (75), (76) and the spiral in the $(u, v)$ plane determine the values of $\alpha$ corresponding to a given temperature or energy. Considering Eq. (75), we find that there is no intersection for $\eta=\frac{\beta G M}{R}>v_{\max }=2.52$. Therefore, below a critical temperature $k T_{\mathrm{c}}=\frac{G m M}{2.52 R}$, an isothermal sphere is expected to collapse. This classical result is not altered by special relativity. Considering now the microcanonical ensemble, we first note that Eq. (76) can be rewritten

$u_{0}=3-\mu v_{0} \mathcal{F}\left(\mu v_{0}\right)-\Lambda v_{0}$.

In the classical limit $(\mu \rightarrow+\infty)$ it reduces to the straight line found by Padmanabhan (1989)

$u_{0}=\frac{3}{2}-\Lambda v_{0}$,

and in the limit $\mu \rightarrow 0$, we find another straight line

$u_{0}=-\Lambda v_{0}$.

The curve (77) is ploted in Fig. 4 for a fixed value of $\mu$ and for different values of $\Lambda$. For $\Lambda>\Lambda_{\mathrm{c}}(\mu)$ there is no intersection, for $\Lambda=\Lambda_{\mathrm{c}}(\mu)$ the curve (77) is tangent to the spiral and for $\Lambda<\Lambda_{\mathrm{c}}(\mu)$ there are one or several intersections. We recover therefore by this graphical construction the existence of a critical energy below which no hydrostatic equilibrium can exist for isothermal spheres. In Fig. 5, we plot the same diagram as Fig. 4 but for different values of $\mu$ and, in each case, for the critical energy $\Lambda_{\mathrm{c}}(\mu)$. The intersection with the spiral determines the value of $\alpha$ at the critical point. This figure confirms that instability occurs sooner (i.e., for smaller values of $\alpha$ or smaller density contrasts) when relativistic effects are taken into account.

\subsection{The condition of thermodynamical stability}

We now address the question of thermodynamical stability. Let us recall that the isothermal spheres lying on the spirals of Fig. 1 are critical points of entropy but they are not necessarily entropy maxima. To determine whether they are local entropy maxima or saddle points, we must examine the sign of the second variations of entropy given by Eq. (37). In fact, this condition of stability has the same form as in the classical case provided that we make the substitution

$$
-\frac{1}{3 N k T^{2}} \rightarrow \frac{k}{2 M m c^{4}} \frac{1}{\mathcal{F}^{\prime}(x)} .
$$

Therefore, the analysis of Padmanabhan (1989) for the classical Antonov instability can be extended straightforwardly. Introducing the mass perturbation $q(r) \equiv$ $\delta M(r)=\int_{0}^{r} 4 \pi r^{\prime} \delta \rho\left(r^{\prime}\right) \mathrm{d} r^{\prime}$ within the sphere of radius $r$ such that

$\delta \rho=\frac{1}{4 \pi r^{2}} \frac{\mathrm{d} q}{\mathrm{~d} r}$

the second variations of entropy can be put in a quadratic form

$\delta^{2} S=\int_{0}^{R} \int_{0}^{R} \mathrm{~d} r \mathrm{~d} r^{\prime} q(r) K\left(r, r^{\prime}\right) q\left(r^{\prime}\right)$,

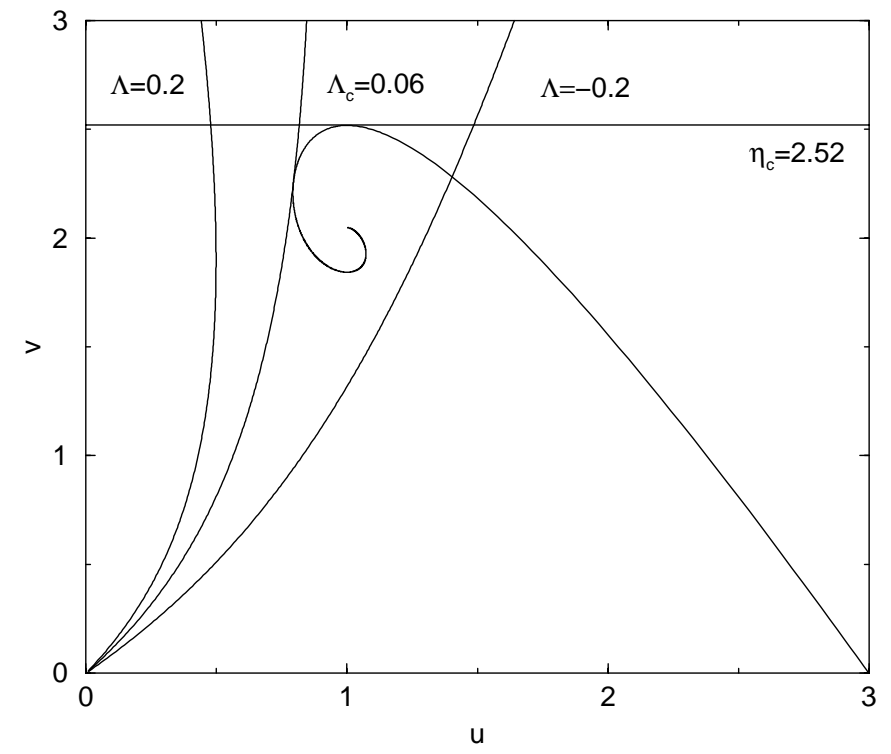

Fig. 4. The $(u, v)$ plane. All isothermal spheres must necessarily lie on the spiral. There exists solutions in the canonical ensemble only for $\eta<2.52$. In the microcanonical ensemble, the critical energy depends on the relativistic parameter $\mu$. For the value $\mu=1$ adopted in the figure, there exists solutions only for $\Lambda<\Lambda_{\mathrm{c}}=0.06$.

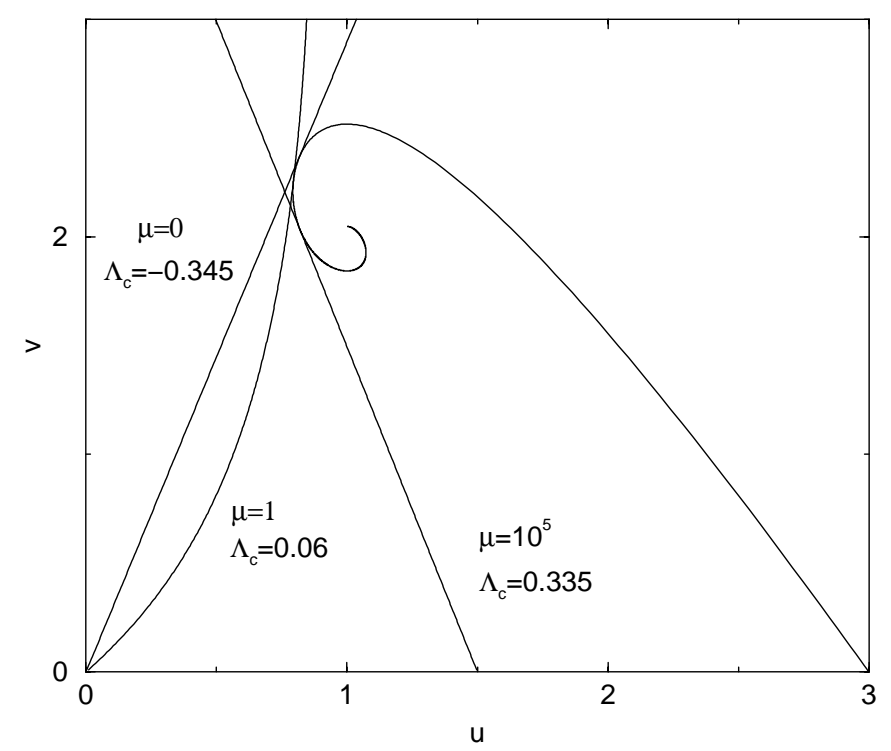

Fig. 5. Same as Fig. 4 but for different values of $\mu$ and, in each case, for the critical parameter $\Lambda_{\mathrm{c}}(\mu)$ above which there is no equilibrium solution.

with

$$
\begin{aligned}
K\left(r, r^{\prime}\right)= & \frac{k}{2 M m c^{4}} \frac{1}{\mathcal{F}^{\prime}(x)} \frac{\mathrm{d} \Phi}{\mathrm{d} r}(r) \frac{\mathrm{d} \Phi}{\mathrm{d} r}\left(r^{\prime}\right) \\
& +\frac{1}{2} \delta\left(r-r^{\prime}\right)\left[\frac{G}{T r^{2}}+\frac{k}{m} \frac{\mathrm{d}}{\mathrm{d} r}\left(\frac{1}{4 \pi \rho r^{2}} \frac{\mathrm{d}}{\mathrm{d} r}\right)\right]
\end{aligned}
$$

Clearly, the conservation of mass imposes the boundary conditions $q(0)=q(R)=0$. The problem of stability 
can therefore be reduced to the study of the eigenvalue equation

$\int_{0}^{R} K\left(r, r^{\prime}\right) F_{\lambda}\left(r^{\prime}\right) \mathrm{d} r^{\prime}=\lambda F_{\lambda}(r)$,

with the boundary conditions $F_{\lambda}(0)=F_{\lambda}(R)=0$. If all the eigenvalues are negative, then $\delta^{2} S<0$ and the critical point is a local entropy maximum. If one eigenvalue is positive, the critical point is an unstable saddle point. The point of marginal stability is determined by the condition that the largest eigenvalue is equal to zero $(\lambda=0)$. We thus have to solve the differential equation

$$
\begin{array}{r}
{\left[\frac{k}{m} \frac{\mathrm{d}}{\mathrm{d} r}\left(\frac{1}{4 \pi \rho r^{2}} \frac{\mathrm{d}}{\mathrm{d} r}\right)+\frac{G}{T r^{2}}\right] F(r)} \\
\quad=-\frac{k}{M m c^{4}} \frac{1}{\mathcal{F}^{\prime}(x)} V \frac{\mathrm{d} \Phi}{\mathrm{d} r}(r),
\end{array}
$$

with

$V=\int_{0}^{R} \frac{\mathrm{d} \Phi}{\mathrm{d} r}\left(r^{\prime}\right) F\left(r^{\prime}\right) \mathrm{d} r^{\prime}$,

and $F(0)=F(R)=0$. Introducing the dimensionless variables defined in Sect. 2.4, it can be rewritten

$\left[\frac{\mathrm{d}}{\mathrm{d} \xi}\left(\frac{\mathrm{e}^{\psi}}{\xi^{2}} \frac{\mathrm{d}}{\mathrm{d} \xi}\right)+\frac{1}{\xi^{2}}\right] F(\xi)=\chi \frac{\mathrm{d} \psi}{\mathrm{d} \xi}$,

with

$\chi=-\frac{1}{x^{2} \mathcal{F}^{\prime}(x)} \frac{1}{\alpha^{2} \psi^{\prime}(\alpha)} \int_{0}^{\alpha} \frac{\mathrm{d} \psi}{\mathrm{d} \xi}\left(\xi^{\prime}\right) F\left(\xi^{\prime}\right) \mathrm{d} \xi^{\prime}$,

and $F(0)=F(\alpha)=0$. As shown by Padmanabhan (1989), the solutions of the differential equation (87) can be expressed in terms of the solutions of the Emden Eq. (52) as

$F(\xi)=\chi\left[\frac{1}{1-u_{0}}\left(\xi^{3} \mathrm{e}^{-\psi}-\xi^{2} \psi^{\prime}\right)+\xi^{2} \psi^{\prime}\right]$.

We can check that this function satisfies the boundary conditions $F(0)=F(\alpha)=0$. The point of marginal stability is obtained by substituting the solution (89) in Eq. (88). The integrations can be carried out and the solutions expressed in terms of the Milne variables $u_{0}$ and $v_{0}$ (see Padmanabhan 1989 for more details). In our semirelativistic treatment, we obtain

$2 u_{0}^{2}+u_{0} v_{0}-7 u_{0}+3-\mu^{2} v_{0}^{2} \mathcal{F}^{\prime}\left(\mu v_{0}\right)\left(u_{0}-1\right)=0$,

where we have used Eqs. (64) and (75) to simplify the last term.

In the classical limit $\mu \rightarrow+\infty$ we recover the result of Padmanabhan (1989)

$4 u_{0}^{2}+2 u_{0} v_{0}-11 u_{0}+3=0$,

and in the formal limit $\mu \rightarrow 0$, we find

$2 u_{0}+v_{0}-4=0$.

The intersections between the curve (90) and the spiral in the $(u, v)$ plane determine the values of $\alpha$ for which

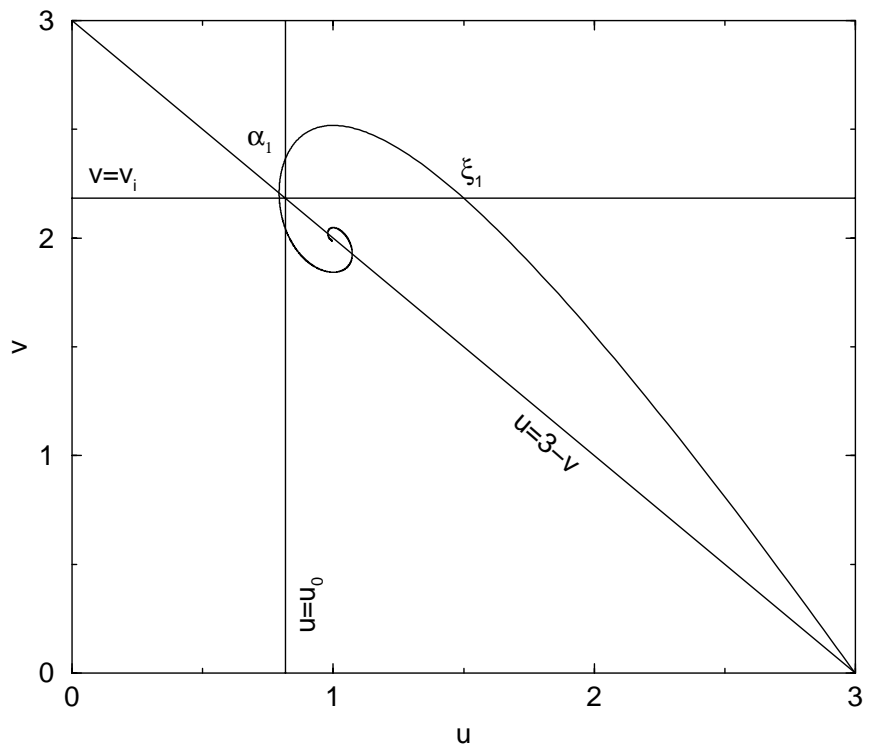

Fig. 6. Graphical construction to determine the nodes of the perturbation profile $\delta \rho$ at the point of marginal stability. The construction is done explicitly for $\mu=0$ (formally), for which $\alpha_{1}=16.0$ and $u_{0}=0.817$. There is only one zero satisfying $\xi_{1}<\alpha_{1}$ so that the perturbation profile does not present a "core-halo" structure. This property is maintained until $\mu>1.61$.

a new mode of stability is lost (i.e., a new eigenvalue $\lambda$ becomes positive). Since the curve (90) passes through the singular sphere $\left(u_{\mathrm{s}}, v_{\mathrm{s}}\right)=(1,2)$ at the center of the spiral, there is an infinity of intersections. The first intersection (for which $\alpha$ is minimum) corresponds to the point of marginal stability denoted $\alpha_{1}$. We can show that the points determined by Eq. (90) are precisely those for which $\Lambda$ is extremum in agreement with the turning point analysis of Katz (1978). Indeed, differentiating the expression (76) for $\Lambda$ with respect to $\alpha$, we get

$\frac{\mathrm{d} \Lambda}{\mathrm{d} \alpha}=-\mu^{2} \mathcal{F}^{\prime}\left(\mu v_{0}\right) \frac{\mathrm{d} v_{0}}{\mathrm{~d} \alpha}-\frac{3}{v_{0}^{2}} \frac{\mathrm{d} v_{0}}{\mathrm{~d} \alpha}-\frac{1}{v_{0}} \frac{\mathrm{d} u_{0}}{\mathrm{~d} \alpha}+\frac{u_{0}}{v_{0}^{2}} \frac{\mathrm{d} v_{0}}{\mathrm{~d} \alpha}$.

Using Eqs. (72), (73), we obtain

$\frac{\mathrm{d} \Lambda}{\mathrm{d} \alpha}=\frac{1}{\alpha v_{0}}\left[2 u_{0}^{2}+u_{0} v_{0}-7 u_{0}+3-\mu^{2} v_{0}^{2} \mathcal{F}^{\prime}\left(\mu v_{0}\right)\left(u_{0}-1\right)\right]$

and we check that the condition $\frac{\mathrm{d} \Lambda}{\mathrm{d} \alpha}=0$ is equivalent to Eq. (90).

It is also easy to determine the form of the perturbation that triggers the instability at the critical point $\Lambda_{\mathrm{c}}(\mu)$. According to Eq. (81), the eigenfunction associated with the eigenvalue $\lambda=0$ can be written

$\frac{\delta \rho}{\rho_{0}}=\frac{1}{4 \pi \xi^{2}} \frac{\mathrm{d} F}{\mathrm{~d} \xi}$,

where $F(\xi)$ is given by Eq. (89). Simplifying the derivative with the aid of Eq. (52), we can express the perturbation profile in terms of the Milne variables (71) as

$\frac{\delta \rho}{\rho}=\frac{\chi}{4 \pi} \frac{1}{1-u_{0}}\left(3-v-u_{0}\right)$. 


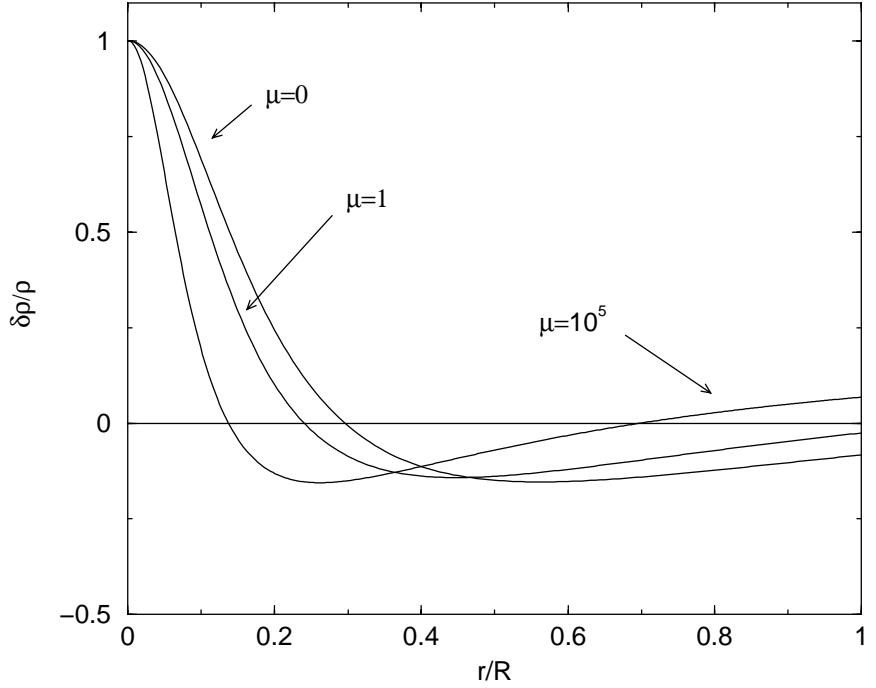

Fig. 7. Density perturbation profile at the point of marginal stability as a function of the relativistic parameter.

The qualitative behavior of the perturbation profile can be studied without numerical integration by a graphical construction (see Padmanabhan 1989). The density perturbation $\delta \rho$ becomes zero at the point(s) $\xi_{i}$ such that $u_{0}=3-v\left(\xi_{i}\right)$. In Fig. 6, we first draw the line $u=3-v$. This line passes through the singular sphere $\left(u_{\mathrm{s}}=1\right.$, $v_{\mathrm{s}}=2$ ) and also cuts the spiral at the points of vertical tangent (see Eq. (72)). In particular, the first intersection corresponds to $\alpha_{*}=22.5$ and $\left(u_{*}, v_{*}\right)=(0.793,2.208)$. Then, we draw the line $u=u_{0}=u\left(\alpha_{1}\right)$. The intersection between these two lines determines $v\left(\xi_{i}\right)$. The intersection between $v=v\left(\xi_{i}\right)$ and the spiral determines the zeros of $\delta \rho$. For $\alpha_{1}>\alpha_{*}$, there are two intersections satisfying $\xi_{i}<\alpha_{1}$ so that the perturbation profile presents a "core-halo" structure. This is the case in particular in the classical limit $\mu \rightarrow+\infty$ for which $\alpha_{1}=34.4$ (see Padmanabhan 1989). By contrast, for $\alpha_{1}<\alpha_{*}$, there is only one intersection satisfying $\xi_{1}<\alpha_{1}$ so that the perturbation profile does not present a "core-halo" structure. This is the case in particular in the (formal) limit $\mu=0$ for which $\alpha_{1}=16.0$. The "core-halo" structure disappears for $\alpha_{1}=\alpha_{*}=22.5$ corresponding to a relativistic parameter $\mu_{*}=1.61$. Since our study is valid for $\mu \gg 1$, we deduce that the density perturbation profile always presents a "core-halo" structure in the cases of physical interest. However, relativistic effects have the tendency to reduce the extent of the halo (see Fig. 7).

The previous results are valid in the microcanonical ensemble in which the energy is fixed. In the canonical ensemble, we must consider maxima of the free energy $J=S-\beta E$ at fixed temperature. In that case, the condition of stability is given by Eq. (85) with $V=0$. Since the relativistic function $\mathcal{F}(x)$ does not appear anymore in the equations, we conclude that special relativity does not change the classical results in the canonical ensemble. In particular, the perturbation profile does not present a "core-halo" structure at the critical point $\eta_{\mathrm{c}}$ (Chavanis 2001).

\section{Isothermal gaseous spheres in general relativity}

\subsection{The equations governing equilibrium}

We now address the structure and the stability of isothermal gas spheres in the context of general relativity. The Einstein field equations of general relativity are expressed as

$R_{\mu \nu}-\frac{1}{2} g_{\mu \nu} R=-\frac{8 \pi G}{c^{4}} T_{\mu \nu}$

where $R_{\mu \nu}$ is the Ricci tensor, $T_{\mu \nu}$ the energy-momentum tensor and $g_{\mu \nu}$ the metric tensor defined by

$\mathrm{d} s^{2}=-g_{\mu \nu} \mathrm{d} x^{\mu} \mathrm{d} x^{\nu}$,

where $\mathrm{d} s$ is the invariant interval between two neighbouring space-time events.

In the following, we shall restrict ourselves to spherically symmetric systems with motions, if any, only in the radial directions. Under these assumptions, the metric can be written in the form

$\mathrm{d} s^{2}=\mathrm{e}^{\nu} \mathrm{d} \tau^{2}-r^{2}\left(\mathrm{~d} \theta^{2}+\sin ^{2} \theta \mathrm{d} \phi^{2}\right)-\mathrm{e}^{\lambda} \mathrm{d} r^{2}, \quad \tau=\mathrm{ct}$,

where $\nu$ and $\lambda$ are functions of $r$ and $\tau$ only. The energymomentum tensor is assumed to be that for a perfect fluid

$T^{\mu \nu}=p g^{\mu \nu}+(p+\epsilon) u^{\mu} u^{\nu}$,

where $u^{\mu}=\mathrm{d} x^{\mu} / \mathrm{d} s$ is the fluid four-velocity, $p$ is the isotropic pressure and $\epsilon$ is the energy density including the rest mass.

The equations of general relativity governing the hydrostatic equilibrium of a spherical distribution of matter are well known. They are given by (see, e.g., Weinberg 1972)

$\frac{\mathrm{d}}{\mathrm{d} r}\left(r \mathrm{e}^{-\lambda}\right)=1-\frac{8 \pi G}{c^{4}} r^{2} \epsilon$,

$\frac{\mathrm{d} p}{\mathrm{~d} r}=-\frac{1}{2}(\epsilon+p) \frac{\mathrm{d} \nu}{\mathrm{d} r}$

$\frac{\mathrm{e}^{-\lambda}}{r} \frac{\mathrm{d} \nu}{\mathrm{d} r}=\frac{1}{r^{2}}\left(1-\mathrm{e}^{-\lambda}\right)+\frac{8 \pi G}{c^{4}} p$.

These equations can be deduced from the Einstein Eq. (97). However, Eq. (102) can be obtained more directly from the local law of energy-momentum conservation, $D_{\mu} T^{\mu \nu}=0$, which is also contained in the Einstein equations.

Equations (101)-(103) can be combined to give

$\left\{1-\frac{2 G M(r)}{c^{2} r}\right\} \frac{\mathrm{d} p}{\mathrm{~d} r}=-\frac{1}{c^{2}}(\epsilon+p)\left\{\frac{G M(r)}{r^{2}}+\frac{4 \pi G}{c^{2}} p r\right\}$

and

$M(r)=\frac{4 \pi}{c^{2}} \int_{0}^{r} \epsilon r^{2} \mathrm{~d} r$. 
These equations are known as the Oppenheimer-Volkoff equations. They extend the classical condition of hydrostatic equilibrium for a star to the context of general relativity. Using Eqs. (101) and (103), we find that the metric functions $\lambda(r)$ and $\nu(r)$ satisfy the relations

$\mathrm{e}^{-\lambda}=1-\frac{2 G}{r c^{2}} M(r)$

and

$\frac{\mathrm{d} \nu}{\mathrm{d} r}=\frac{1+4 \pi p r^{3} / M(r) c^{2}}{r\left(r c^{2} / 2 G M(r)-1\right)}$.

In the empty space outside the star, $p=\epsilon=0$. Therefore, if $M=M(R)$ denotes the total mass-energy of the star, Eqs. (106), (107) become for $r>R$

$\mathrm{e}^{-\lambda}=1-\frac{2 G M}{r c^{2}}, \quad \frac{\mathrm{d} \nu}{\mathrm{d} r}=\frac{1}{r\left(r c^{2} / 2 G M-1\right)}$.

The second equation is readily integrated in

$\nu=\ln \left(1-2 G M / r c^{2}\right)$,

where we have taken the constant of integration to be zero by convention. Substituting the foregoing expressions for $\lambda$ and $\nu$ in Eq. (99), we obtain the well-known Schwarzschild's form of the metric outside a star

$$
\begin{array}{r}
\mathrm{d} s^{2}=\left(1-\frac{2 G M}{r c^{2}}\right) \mathrm{d} \tau^{2}-r^{2}\left(\mathrm{~d} \theta^{2}+\sin ^{2} \theta \mathrm{d} \phi^{2}\right) \\
-\frac{\mathrm{d} r^{2}}{1-2 G M / r c^{2}} .
\end{array}
$$

This metric is singular at

$r=\frac{2 G M}{c^{2}} \equiv R_{\mathrm{S}}$,

where $R_{\mathrm{S}}$ is the Schwazschild radius appropriate to the mass $M$. This does not mean that spacetime is singular at that radius but only that this particular metric is. Indeed, the singularity can be removed by a judicious change of coordinate system (see, e.g., Weinberg 1972). When $R_{\mathrm{S}}>R$, the star is a black hole and no particle or even light can leave the region $R<r<R_{\mathrm{S}}$. However, in our case the discussion does not arise because $R_{\mathrm{S}}<R$. Indeed, for a gaseous sphere in hydrostatic equilibrium, it can be shown that the radius of the configuration is necessarily restricted by the inequality (Buchdahl 1959)

$R \geq \frac{9}{8} \frac{2 G M}{c^{2}}=\frac{9}{8} R_{\mathrm{S}}$

Therefore, the points exterior to the star always satisfy $r>R_{\mathrm{S}}$.

\subsection{The equation of state}

To close the system of Eqs. (104), (105), we need to specify an equation of state relating the pressure $p$ to the energy density $\epsilon$. Quite generally, the first law of thermodynamics can be expressed as

$\mathrm{d}\left(\frac{\epsilon}{n}\right)=-p \mathrm{~d}\left(\frac{1}{n}\right)+T \mathrm{~d} s$

or, equivalently,

$\mathrm{d} \epsilon=\frac{p+\epsilon}{n} \mathrm{~d} n+n T \mathrm{~d} s$,

where $n$ is the baryon number density and $s$ the entropy per baryon in rest frame. It must be completed by two equations of state $p=p(n, s)$ and $T=T(n, s)$. Then, Eq. (114) can be integrated to give $\epsilon(n, s)$ (see, e.g., Misner et al. 1973).

We shall assume in the following that the term $T \mathrm{~d} s$ in Eq. (114) can be neglected. This simplification arises in two different situations. In the case of neutron stars or white dwarfs, the thermal energy $k T$ is much smaller than the Fermi energy, so the neutrons or the electrons are completely degenerate $\left(k T \ll E_{\text {fermi }}\right)$. On the other hand, in supermassive stars, temperature and entropy are important but convection keeps the star stired up and produces a uniform entropy distribution ( $s=$ const.). In these two important situations, the first law of thermodynamics reduces to

$\mathrm{d} \epsilon=\frac{p+\epsilon}{n} \mathrm{~d} n$,

and we just require one equation of state $p=p(n)$. We shall consider an equation of state of the form

$p=K n^{\gamma}$,

where $K$ and $\gamma$ are constant. It is easy to check that the general solution of Eq. (115) with Eq. (116) is

$\epsilon=A p^{1 / \gamma}+\frac{1}{\gamma-1} p$,

where $A$ is a constant. Systems obeying the pressureenergy density relation (117) with $A \neq 0$ have been considered by Tooper (1965). Since the relation between $\epsilon$ and $p$ is essentially a power-law, these systems generalize the polytropes of Newtonian theory. We shall here consider the limiting situation $A=0$. In that case, Eq. (117) reduces to the so-called "gamma law" equation of state

$p=q \epsilon \quad$ with $\quad q=\gamma-1$.

Since the relation between $p$ and $\epsilon$ is linear, this equation of state extends the theory of isothermal spheres to the context of general relativity. As noted by Chandrasekhar (1972), for this equation of state, the Oppenheimer-Volkoff equations become mathematically similar to those describing a classical isothermal gas (the so-called Emden equations).

An equation of state of the form (118) has been introduced in different situations:

(i) This equation of state prevails in the highly energetic core of neutron stars where the matter is ultra-relativistic and completely degenerate 
(Oppenheimer \& Volkoff 1939; Misner \& Zapolsky 1964; Meltzer \& Thorne 1966). If the system is modelled as a pure collection of noninteracting fermions, standard theory leads to (Chandraskhar 1942)

$p=\frac{1}{3} \epsilon \quad(\gamma=4 / 3)$.

In that case, the constant $K$ which appears in Eq. (116) is explicitly given by $K=\frac{1}{8}\left(\frac{3}{\pi}\right)^{1 / 3} h c$, where $h$ is the Planck constant. Other versions of the equation of state have attempted to take into account nucleon-nucleon interactions or a spectrum of baryon species. Equations of the form (118) but with numerical coefficient other than $1 / 3$ have sometimes been suggested. For example, Ambartsumyan \& Saakyan (1961) have used an equation with $q=1 / 13$ $(\gamma=14 / 13)$ and Tsuruta \& Cameron (1966) with $q=1$. The equation of state

$p=\epsilon$,

was also introduced by Zel'dovich (1962) for a gas of baryons interacting through a vector meson field. This represents an extremely high pressure since the sound velocity, $(\mathrm{d} p / \mathrm{d} \epsilon)^{1 / 2} c$, is equal to the velocity of light for this value of $q$. This is clearly an upper bound. In the following, we shall consider a general equation of state of the form (118) with $q$ in the range $0 \leq q \leq 1$.

(ii) Bisnovatyi-Kogan \& Zel'dovich (1969) and Bisnovatyi-Kogan \& Thorne (1970) have considered the general relativistic equilibrium configuration of a gas whose temperature $T$ is constant everywhere. In that case, the equation of state is given by (see Sect. 2)

$p=\frac{K_{2}\left(\frac{m c^{2}}{k T}\right)}{\frac{m c^{2}}{k T} K_{3}\left(\frac{m c^{2}}{k T}\right)-K_{2}\left(\frac{m c^{2}}{k T}\right)} \epsilon$,

which is again of the form (118). In particular, $q=$ $\frac{1}{3}$ when $T \rightarrow+\infty$. However, as Bisnovatyi-Kogan \& Thorne emphasize, the equation of state (121) can only describe a "local" thermodynamical equilibrium. Indeed, "global" thermodynamical equilibrium in general relativity requires that the redshifted temperature $\mathrm{e}^{\nu(r) / 2} T$ be uniform throughout the medium (Tolman 1934). Thus, the spheres described by Eq. (121) are not "isothermal" in the general-relativistic sense; heat will slowly diffuse inward in them, upsetting the condition $T=$ const. and trying to establish $\mathrm{e}^{\nu(r) / 2} T(r)=$ const. instead. However, since the hydrostatic Eqs. (104), (105) with the equation of state (118) are mathematically similar to the classical Emden equation for isothermal gas spheres, we shall call the equation of state (118) "isothermal", following the terminology of Chandrasekhar (1972), although this is only correct in a local sense.

(iii) The equation of state (118) was also proposed by Saslaw et al. (1996) in a cosmological context to model the ultimate state of an Einstein-de Sitter universe that undergoes a phase transition caused by gravitational clustering. This phase transition can lead to the growth of a centrally concentrated distribution of matter so that the universe would pass from a statistically homogeneous state to a state of rotational symmetry around one point only. The resulting configuration neither expands nor contracts, so the global solution is stationary. According to Saslaw et al. (1996), this "isothermal universe" would represent the ultimate astrophysical prediction.

(iv) Note finally that the equation of state $p=\frac{1}{3} \epsilon$ prevails in a medium where the pressure is entirely due to radiation (Chandrasekhar 1942).

\subsection{The general relativistic Emden equation}

Considering the equation of state (118), we shall introduce the dimensionless variables $\xi, \psi$ and $M(\xi)$ by the relations

$\epsilon=\epsilon_{0} \mathrm{e}^{-\psi}, \quad r=\left\{\frac{c^{4} q}{4 \pi G \epsilon_{0}(1+q)}\right\}^{1 / 2} \xi$

and

$M(r)=\frac{4 \pi \epsilon_{0}}{c^{2}}\left\{\frac{c^{4} q}{4 \pi G \epsilon_{0}(1+q)}\right\}^{3 / 2} M(\xi)$.

In terms of the variables $\psi$ and $\xi$, Eqs. (104) and (105) can be reduced to the following dimensionless forms (Chandrasekhar 1972)

$\left\{1-\frac{2 q}{1+q} \frac{M(\xi)}{\xi}\right\} \frac{\mathrm{d} \psi}{\mathrm{d} \xi}=\frac{M(\xi)}{\xi^{2}}+q \xi \mathrm{e}^{-\psi}$

and

$\frac{\mathrm{d} M(\xi)}{\mathrm{d} \xi}=\xi^{2} \mathrm{e}^{-\psi}$

In addition, the metric functions determined by Eqs. (106) and (102) can be expressed as

$\mathrm{e}^{-\lambda}=1-\frac{2 q}{1+q} \frac{M(\xi)}{\xi}, \quad \nu=\frac{2 q}{1+q} \psi(\xi)+$ const.,

where the constant is determined by the matching with the outer Schwarzschild solution (109) at $r=R$.

The Newtonian limit corresponds to $q \rightarrow 0$. In that limit, Eqs. (124), (125) reduce to

$\frac{\mathrm{d} \psi}{\mathrm{d} \xi}=\frac{M(\xi)}{\xi^{2}}, \quad$ and $\quad \frac{\mathrm{d} M(\xi)}{\mathrm{d} \xi}=\xi^{2} \mathrm{e}^{-\psi}$,

and they combine to give Emden's equation

$\frac{1}{\xi^{2}} \frac{\mathrm{d}}{\mathrm{d} \xi}\left(\xi^{2} \frac{\mathrm{d} \psi}{\mathrm{d} \xi}\right)=\mathrm{e}^{-\psi}$

Therefore, Eqs. (124), (125) represent the general relativistic equivalent of the Emden equation. Like in the Newtonian case, they admit a singular solution of the form (Chandrasekhar 1972)

$\mathrm{e}^{-\psi_{\mathrm{s}}}=\frac{Q}{\xi^{2}}, \quad$ where $\quad Q=\frac{2(1+q)}{(1+q)^{2}+4 q}$.

The metric associated with the singular isothermal sphere is given explicitly by

$\mathrm{e}^{\nu}=A \xi^{\frac{4 q}{1+q}}, \quad \mathrm{e}^{\lambda}=1+\frac{4 q}{(1+q)^{2}}$, 
where $A$ is an unimportant constant. Considering now the regular solutions of Eqs. (124), (125), we can always suppose that $\epsilon_{0}$ represents the energy density at the center of the configuration. Then, Eqs. (124), (125) must be solved with the boundary conditions

$\psi(0)=\psi^{\prime}(0)=0$.

The corresponding solution must be computed numerically. However, it is possible to determine its asymptotic behaviors explicitly. For $\xi \rightarrow 0$,

$\psi=a \xi^{2}+b \xi^{4}+\ldots$,

with

$a=\frac{1+3 q}{6}, \quad b=-\frac{\left(15 q^{2}-2 q+3\right)(1+3 q)}{360(1+q)}$,

and for $\xi \rightarrow+\infty$ (Chandrasekhar 1972)

$$
\begin{aligned}
\mathrm{e}^{-\psi}= & \frac{Q}{\xi^{2}}\left\{1+\frac{A}{\xi^{(1+3 q) / 2(1+q)}}\right. \\
& \left.\times \cos \left(\frac{\left(7+42 q-q^{2}\right)^{1 / 2}}{2(1+q)} \ln \xi+\delta\right)\right\} .
\end{aligned}
$$

The curve (134) intersects the singular solution (129) infinitely often at points that asymptotically increase geometrically in the ratio $1: \exp \frac{2 \pi(1+q)}{\left(7+42 q-q^{2}\right)^{1 / 2}}$ (see Fig. 8). Since the energy density $\epsilon$ falls off as $\xi^{-2}$ at large distances, the total mass-energy $M$ is infinite. In practice, this "infinite mass problem" does not arise because the isothermal equation of state (118) only holds in a finite region of space (e.g., the inner regions of a neutron star). For simplicity, we shall remedy this difficulty by assuming that our system is confined within a box of radius $R$, like in Sect. 2. This is clearly an idealization but it provides a well-posed model which captures the essential features of the system (see below) and which can be be studied in great detail without any further approximation.

If the system is enclosed within a box, the solution of Eqs. (124), (125) must be terminated at a radius $\alpha$ given by

$\alpha=\left\{\frac{4 \pi G \epsilon_{0}(1+q)}{c^{4} q}\right\}^{1 / 2} R$.

It should be noted that $\alpha$ is a measure of the central energy density $\epsilon_{0}$ (for a given box radius $R$ ). Instead of $\alpha$, we might prefer to consider the density contrast

$\mathcal{R} \equiv \frac{\epsilon_{0}}{\epsilon(R)}=\mathrm{e}^{\psi(\alpha)}$.

The density contrast is a monotonous function of $\alpha$ varying from $\mathcal{R}=1$ (homogeneous system, $\alpha=0$ ) to $\mathcal{R} \rightarrow+\infty$ (singular sphere, $\alpha \rightarrow+\infty$ ). It is sometimes of interest to express the results in terms of the redshift (see, e.g., Weinberg 1972)

$z=\frac{\Delta \lambda}{\lambda}=\left|g_{00}(r)\right|^{-1 / 2}-1=\mathrm{e}^{-\nu(r) / 2}-1$.

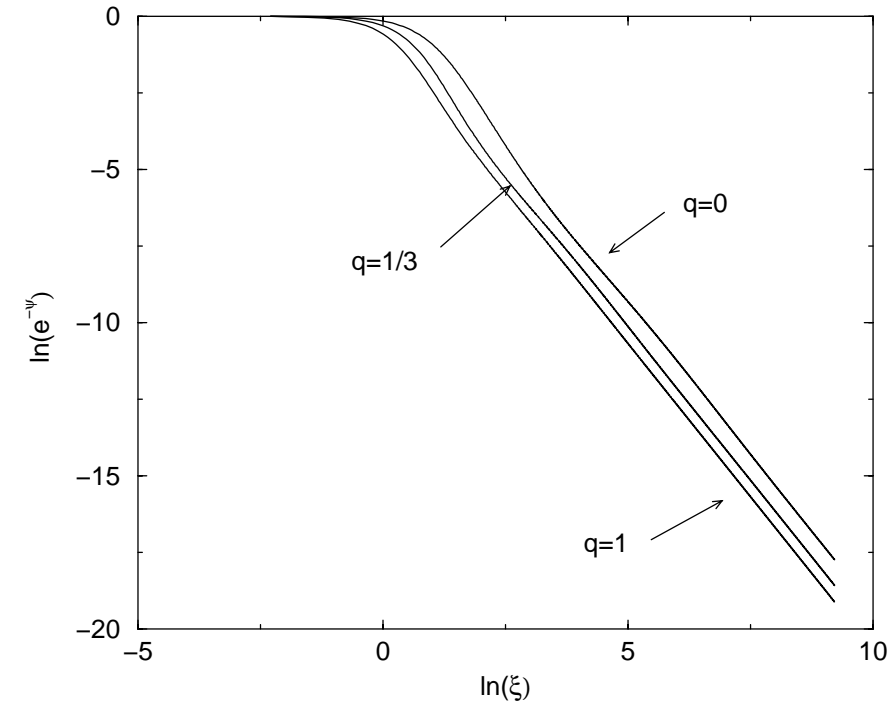

Fig. 8. Density profiles of the general relativistic Emden equation for different values of $q$. The profiles behaves like $\xi^{-2}$ at large distances.

Using Eq. (126) with the boundary condition (109) at $r=R$, the redshift of a spectral line emitted from an isothermal sphere is given by

$z(\xi)=\left(1-\frac{2 G M}{R c^{2}}\right)^{-1 / 2} \exp \left\{\frac{q}{1+q}(\psi(\alpha)-\psi(\xi))\right\}-1$.

\subsection{The Milne variables}

As in the Newtonian theory, it will be convenient in the following to introduce the Milne variables

$u=\frac{\xi \mathrm{e}^{-\psi}}{\psi^{\prime}}, \quad$ and $\quad v=\xi \psi^{\prime}$.

In terms of these variables, the system of Eqs. (124), (125) can be reduced to a single first order differential equation (Chandrasekhar 1972)

$$
\begin{aligned}
& \frac{u}{v} \frac{\mathrm{d} v}{\mathrm{~d} u}= \\
& \quad \frac{-1-\frac{2 q}{1+q} v+(1+3 q) u+\frac{q(3+5 q)}{1+q} u v+\frac{2 q^{2}(1-q)}{(1+q)^{2}} u v^{2}}{3-\frac{1-q}{1+q} v-(1+3 q) u-\frac{q(3+q)}{1+q} u v-\frac{4 q^{2}}{(1+q)^{2}} u v^{2}} .
\end{aligned}
$$

For $\xi \rightarrow 0$, one has

$u=\frac{1}{2 a}-\left(\frac{b}{a^{2}}+\frac{1}{2}\right) \xi^{2}+\ldots, \quad v=2 a \xi^{2}+\ldots$,

and for $\xi \rightarrow+\infty$

$u \rightarrow u_{\mathrm{s}}=\frac{Q}{2}, \quad v \rightarrow v_{\mathrm{s}}=2$.

The solution curve in the $(u, v)$ plane is parametrized by $\xi$. Starting from the point $(u, v)=\left(\frac{3}{1+3 q}, 0\right)$ for $\xi=0$ with a 


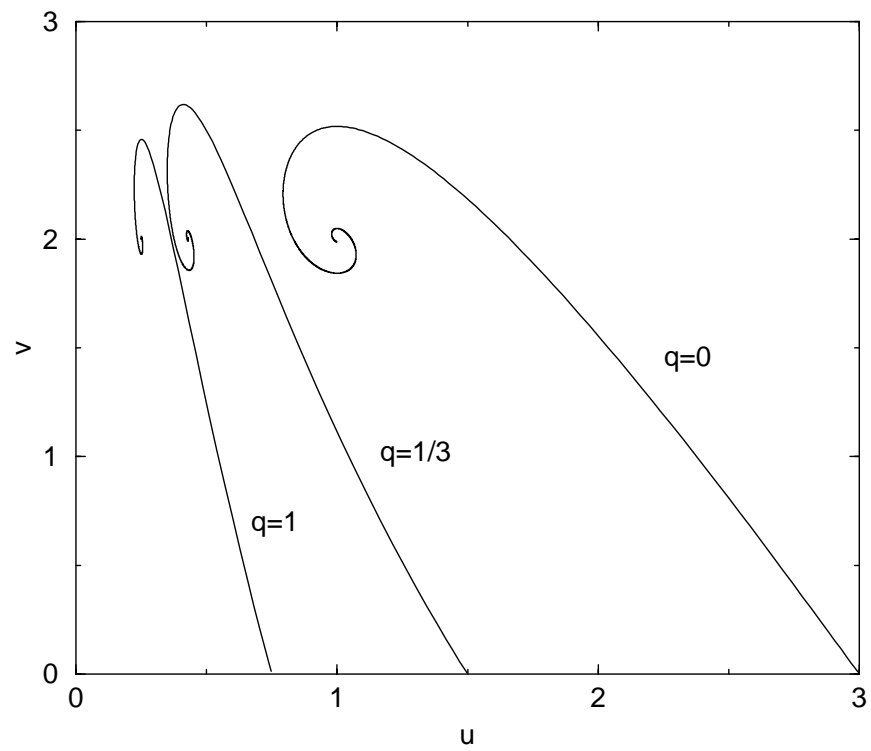

Fig. 9. The $(u, v)$ plane for isothermal gaseous spheres in general relativity and for different values of $q$. The value $q=0$ corresponds to the Newtonian limit.

slope $(\mathrm{d} v / \mathrm{d} u)_{0}=-\frac{4 a^{3}}{a^{2}+2 b}$, the solution curve spirals indefinitely around the point $\left(u_{\mathrm{s}}, v_{\mathrm{s}}\right)=\left(\frac{Q}{2}, 2\right)$, corresponding to the singular sphere, as $\xi \rightarrow+\infty$. All isothermal spheres must necessary lie on this curve (see Fig. 9). For bounded isothermal spheres, $\xi$ must be terminated at the box radius $\alpha$. Clearly, the spiral behavior of the $(u, v)$ curve can be ascribed to the oscillating behavior of the solution (134) as $\xi \rightarrow+\infty$. An explicit parametric equation for the spiral (valid for $\xi \rightarrow+\infty$ ) can be obtained by substituting the asymptotic expansion (134) in the Milne variables (139) like in the Newtonian case (see Chavanis 2001).

\subsection{Oscillatory behavior of the mass-density profile}

According to Eq. (123), the relation between the total mass $M$ of the configuration and the central energy density $\epsilon_{0}$ (through the parameter $\alpha$ ) is given by

$M=\frac{q}{1+q} \frac{M(\alpha)}{\alpha} \frac{R c^{2}}{G}$.

Solving for $M(\xi)$ is Eq. (124), we get

$\frac{M(\alpha)}{\alpha}=\frac{\alpha \psi^{\prime}(\alpha)-q \alpha^{2} \mathrm{e}^{-\psi(\alpha)}}{1+p \alpha \psi^{\prime}(\alpha)}, \quad\left(p=\frac{2 q}{1+q}\right)$.

The relation (143) with Eq. (144) can be expressed very simply in terms of the values of the Milne variables $u$ and $v$ at the normalized box radius $\alpha$. Writing $u_{0}=u(\alpha)$ and $v_{0}=v(\alpha)$ and using Eq. (139), we obtain

$\chi \equiv \frac{2 G M}{R c^{2}}=\frac{p v_{0}\left(1-q u_{0}\right)}{1+p v_{0}}$.

The curve $\chi(\alpha)$ starts from $\chi=0$ for $\alpha=0$ and oscillates around its asymptotic value $\chi_{\mathrm{s}}=p Q=\frac{4 q}{(1+q)^{2}+4 q}$

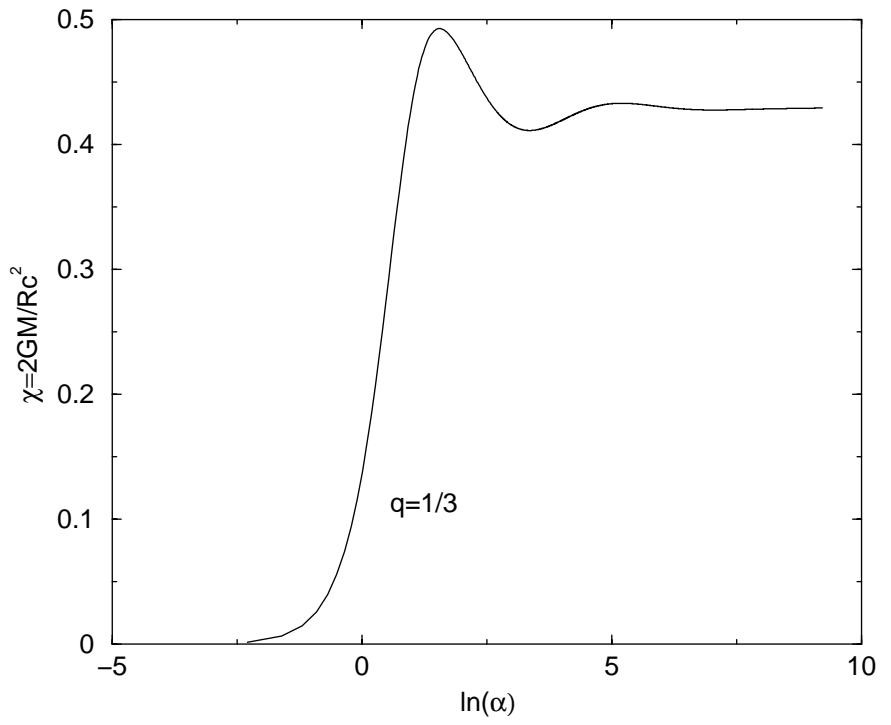

Fig. 10. Total mass-energy $\chi$ versus central energy density $\alpha$ for $q=1 / 3$.

(corresponding to the singular sphere) as $\alpha \rightarrow+\infty$ (see Fig. 10). This relation between the total mass and the central density is very similar to the corresponding one for neutron star models (Oppenheimer \& Volkoff 1939; Misner \& Zapolsky 1964) and dense stellar clusters at statistical equilibrium (Bisnovatyi et al. 1998). In particular, there exists mass peaks in the diagram. In fact, this oscillatory behavior should not cause surprise since the same phenomenon exists for a classical isothermal gas (see in particular Fig. 7 of Chavanis 2001 which can be interpreted as a mass-density relation for a fixed temperature and radius).

From Eqs. (143), (144) and (125), one has

$$
\begin{aligned}
\frac{\mathrm{d} \chi}{\mathrm{d} \alpha} & =p \frac{M^{\prime}(\alpha)}{\alpha}-p \frac{M(\alpha)}{\alpha^{2}} \\
& =p \alpha \mathrm{e}^{-\psi(\alpha)}-p \frac{\psi^{\prime}(\alpha)-q \alpha \mathrm{e}^{-\psi(\alpha)}}{1+p \alpha \psi^{\prime}(\alpha)} .
\end{aligned}
$$

In terms of the Milne variables, it can be rewritten

$\frac{\mathrm{d} \chi}{\mathrm{d} \alpha}=\frac{p}{\alpha}\left(u_{0} v_{0}-\frac{v_{0}\left(1-q u_{0}\right)}{1+p v_{0}}\right)$.

Therefore, the extrema of the curve $\chi(\alpha)$, determined by the condition $\mathrm{d} \chi / \mathrm{d} \alpha=0$, satisfy

$p v_{0}=\frac{1}{u_{0}}-q-1$.

This equation defines a hyperbole in the $(u, v)$ plane. For $u_{0} \rightarrow 0^{+}, v_{0} \sim \frac{1}{p u_{0}} \rightarrow+\infty$ and for $u_{0} \rightarrow+\infty$, $v_{0} \rightarrow-\frac{(q+1)^{2}}{2 q}$. The intersections between this curve and the spiral (see Fig. 11) determine the values of $\alpha$ for which $\chi$ is an extremum. Since the curve (148) passes through the center of the spiral $\left(u_{\mathrm{s}}, v_{\mathrm{s}}\right)$, there is an infinity of intersections, resulting in an infinity of oscillations in Fig. 10. 


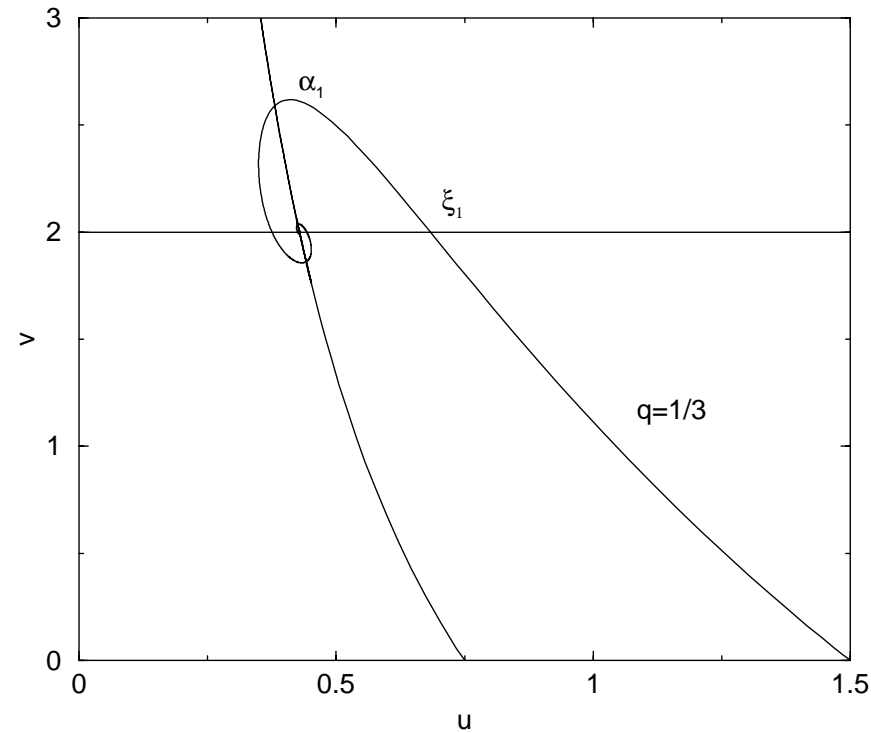

Fig. 11. Graphical construction to determine the critical density $\alpha_{1}$ for $q=1 / 3$.

The first value $\alpha_{1}$ corresponds to the global maximum of $\chi$. Therefore, finite isothermal spheres in general relativity exist only provided that

$\chi \leq \chi_{\mathrm{c}} \equiv \frac{p v\left(\alpha_{1}\right)\left(1-q u\left(\alpha_{1}\right)\right)}{1+p v\left(\alpha_{1}\right)}$.

This implies in particular the existence of a limiting mass (for a given radius $R$ ) such that

$M<M_{\mathrm{c}} \equiv \chi_{\mathrm{c}} \frac{R c^{2}}{2 G}$.

This result is of course related to the limiting mass of neutron stars discovered by Oppenheimer \& Volkoff (1939). In their study, the value of the radius $R$ is determined by a proper modelling of the envelope, instead of an artificial box. However, our results indicate that the essential properties of neutron stars: oscillatory behavior of the massdensity profile, limiting mass and spiral behavior of the mass-radius diagram (see Sect. 3.6) are due primarily to their isothermal core and not to their envelope. Similar observations have been made by Yabushita (1974) who considered the case of an isothermal gaseous sphere surrounded by a medium exerting on it a constant pressure.

The critical parameter $\chi_{\mathrm{c}}$, the critical density contrast $\mathcal{R}_{\mathrm{c}}$ and the critical redshifts $z_{\mathrm{c}}$ emitted from the center and from the boundary of the isothermal configuration are ploted as a function of $q$ in Figs. 12-14. The corresponding values of $\alpha_{1}$ are indicated in Fig. 16 (full line). For the Newtonian case $(q=0)$, we recover the classical values $\alpha_{1}=8.99$ and $\mathcal{R}_{\mathrm{c}}=32.1$ obtained in the canonical ensemble. When $q$ is increased, the critical density $\alpha_{1}$ is lowered so that instability occurs sooner than in the Newtonian case. It should be noted, however, that the critical density contrast $\mathcal{R}_{\mathrm{c}}$ is not a monotonous function of $q$. This is due to the deformation of the spiral in the $(u, v)$ plane

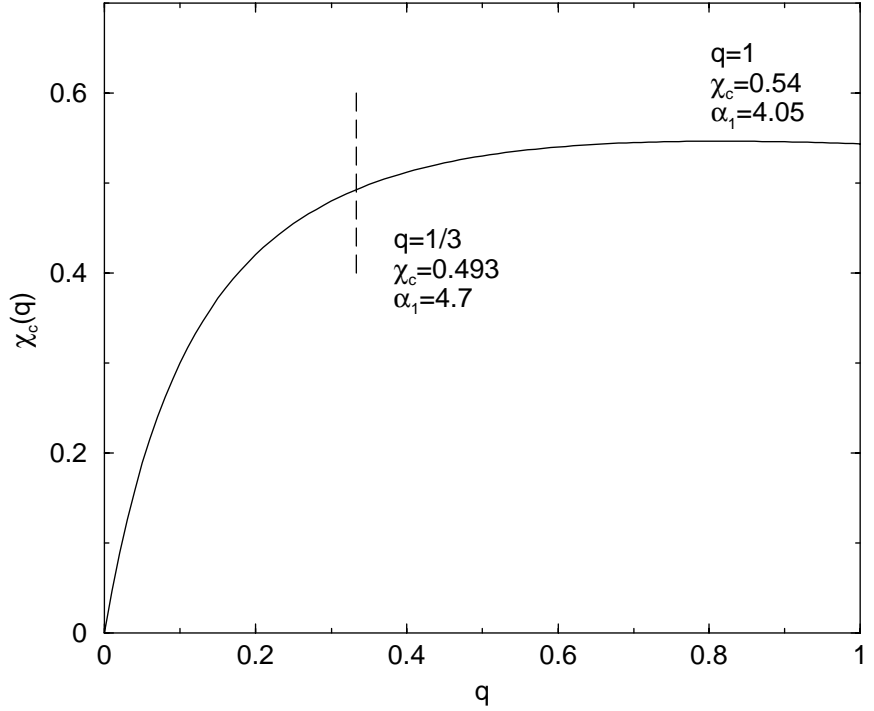

Fig. 12. Critical parameter $\chi_{\mathrm{c}}$ as a function of $q$. The classical limit $q \rightarrow 0$ is discussed in Sect. 4.4.

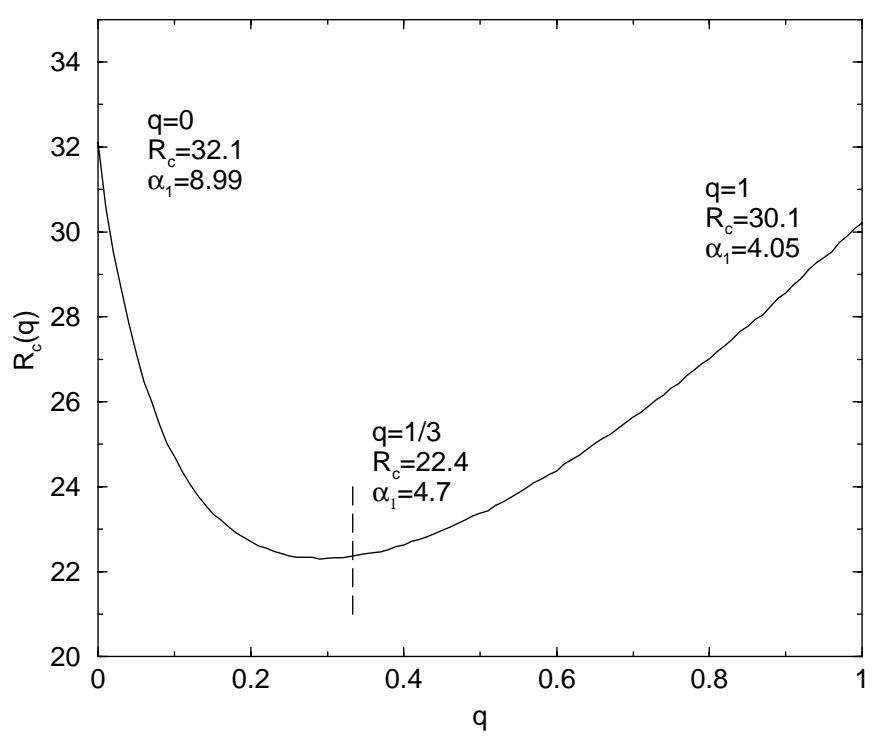

Fig. 13. Critical density contrast $\mathcal{R}_{\mathrm{c}}$ as a function of $q$.

when we vary the relativistic parameter $q$. We find that the value of $q$ for which $\mathcal{R}_{\mathrm{c}}$ is minimum is close to $1 / 3$, the typical value corresponding to neutron stars. However, the variation of the critical density contrast with $q$ is not very important $\left(\mathcal{R}_{\mathrm{c}} \sim 20-30\right.$ in the whole range of parameters $)$ so that the critical central redshift

$z_{0}^{\mathrm{c}}=\left(1-\chi_{\mathrm{c}}\right)^{-1 / 2} \mathcal{R}_{\mathrm{c}}^{\frac{q}{1+q}}-1$

obtained from Eqs. (138), (136), (145), increases monotonically with $q$.

\subsection{The mass-radius diagram}

It is well-known that, for high central densities, the massradius diagram for neutron stars presents a spiral behavior. We show in this section that we can reproduce this 


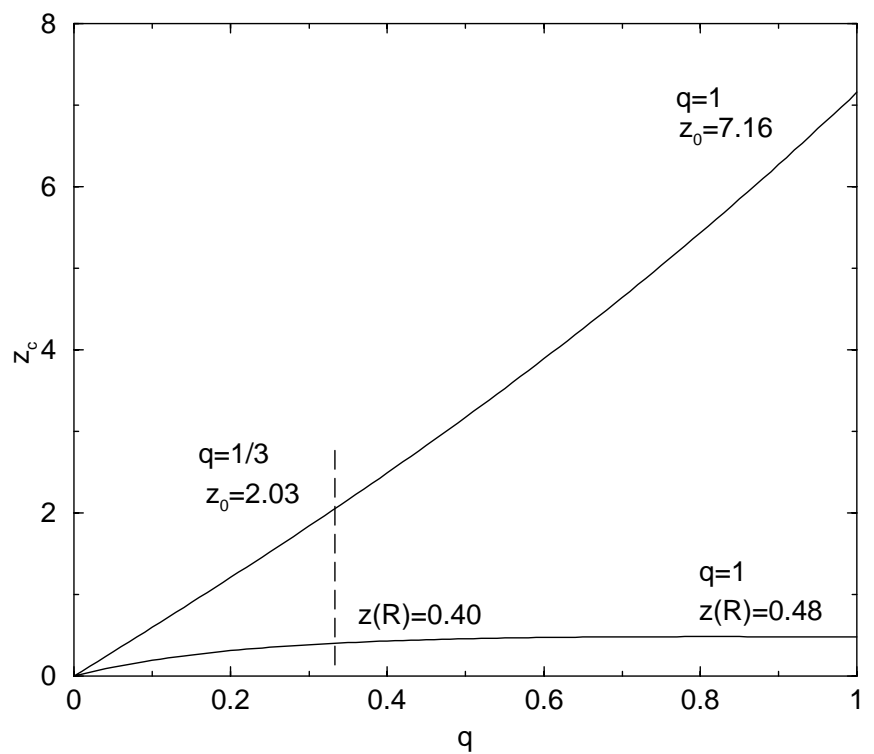

Fig. 14. Critical redshifts $z_{c}$ emitted from the center and from the boundary of an isothermal configuration as a function of $q$.

behavior within our simple "box" model. To that purpose, we shall consider configurations with different masses and radii but with the same density $\epsilon(R)$ at the boundary of the domain. According to Eqs. (135) and (136), we can express the radius $R$ as a function of the parameter $\alpha$ by the relation

$\frac{R}{R_{0}}=\alpha \mathrm{e}^{-\psi(\alpha) / 2}$,

where we have introduced a typical radius

$R_{0}=\left\{\frac{q c^{4}}{4 \pi G \epsilon(R)(1+q)}\right\}^{1 / 2}$.

On the other hand, using Eq. (145), the dimensionless mass appropriate to the present context is given by

$\frac{M}{M_{0}}=\chi(\alpha) \frac{R}{R_{0}}(\alpha)$,

where

$M_{0}=\frac{c^{2} R_{0}}{2 G}$.

Equations (152), (154) determine the mass-radius relation for an isothermal gas with a fixed energy density at its boundary. The $M-R$ curve, represented in Fig. 15, is parametrized by the central density $\alpha$. For comparison with real objects (e.g., neutron stars) we note that the assumption of an isothermal core is valid only for sufficiently large central densities, typically from the point encircled. From that point, our model reproduces qualitatively the spiral behavior of the mass-radius diagram for neutron stars. Using the asymptotic expansion (134) of the function $\psi(\xi)$, it is possible to determine an explicit equation

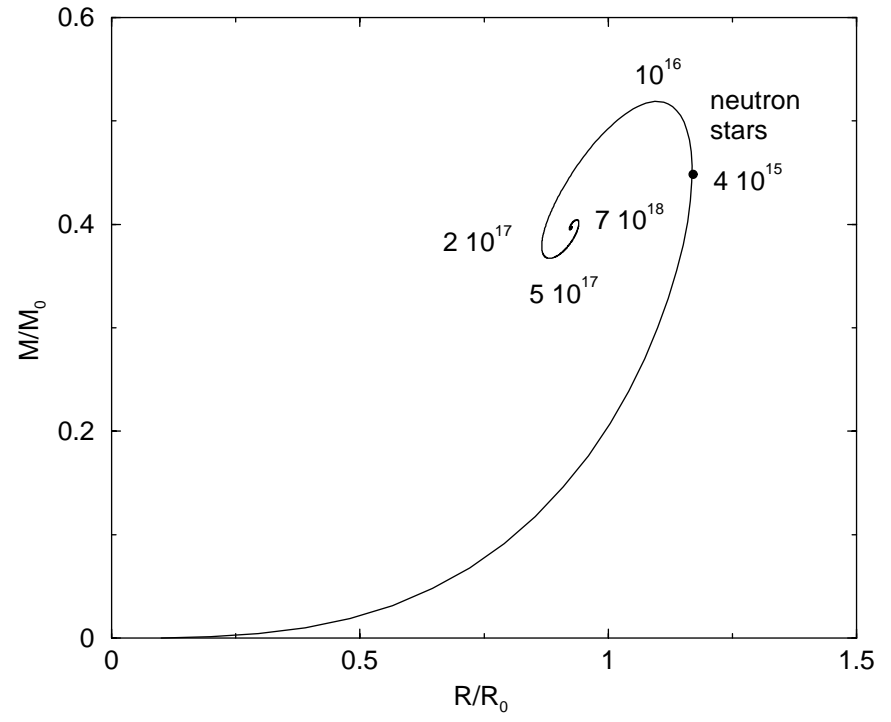

Fig. 15. The mass-radius diagram for an isothermal gas with a fixed energy density at the boundary $\left(\epsilon(R)=10^{15} \mathrm{gc}^{2} / \mathrm{cm}^{3}\right)$. We have indicated the value of the central density $\epsilon_{0}$ at the turning points.

for the spiral (valid for high densities) in the form

$$
\begin{aligned}
& \frac{R}{R_{0}}=Q^{1 / 2}\left\{1+\frac{A}{2 \alpha^{(1+3 q) / 2(1+q)}}\right. \\
& \left.\quad \times \cos \left(\frac{\left(7+42 q-q^{2}\right)^{1 / 2}}{2(1+q)} \ln \alpha+\delta\right)\right\}, \\
& \frac{M}{M_{0}}=p Q^{3 / 2}\left\{1+\frac{A}{4(1+5 q) \alpha^{(1+3 q) / 2(1+q)}}[(1+q)\right. \\
& \quad \times\left(7+42 q-q^{2}\right)^{1 / 2} \sin \left(\frac{\left(7+42 q-q^{2}\right)^{1 / 2}}{2(1+q)} \ln \alpha+\delta\right) \\
& \left.\left.+\left(3+10 q-q^{2}\right) \cos \left(\frac{\left(7+42 q-q^{2}\right)^{1 / 2}}{2(1+q)} \ln \alpha+\delta\right)\right]\right\} .
\end{aligned}
$$

In particular, the terminal point of the spiral, corresponding to the singular solution (129), is given by

$\frac{R_{\mathrm{s}}}{R_{0}}=Q^{1 / 2}, \quad \frac{M_{\mathrm{s}}}{M_{0}}=p Q^{3 / 2}$.

The previous results are valid for any value of the parameter $q$. We shall now specialize on the case of neutron stars for which $q=1 / 3$. For estimating $\epsilon(R)$, we shall adopt the typical value given by Misner \& Zapolsky (1964)

$\epsilon(R)=10^{15} \mathrm{gc}^{2} / \mathrm{cm}^{3}$,

which corresponds in an ideal neutron gas to a Fermi energy of $130 \mathrm{MeV}$. This is the typical value of the energy density in the regime where the kinetic energy of the neutrons is of the same order as their rest mass. This regime corresponds to the transition between the isothermal core and the envelope and it is therefore relevant to adopt this value at $r=R$. For this density,

$R_{0}=5.2 \mathrm{~km}, \quad M_{0}=1.8 M_{\odot}$. 
From the diagram of Fig. 15, we find that the maximum mass and the corresponding radius have the values

$R_{\mathrm{c}}=1.1 R_{0}, \quad M_{\mathrm{c}}=0.5 M_{0}$.

The value of the maximum mass in our model $M_{\mathrm{c}} \sim$ $0.9 M_{\odot}$ is close to the more exact value $\sim 0.7 M_{\odot}$ found by Oppenheimer \& Volkoff (1939) by introducing a more general equation of state also valid in the envelope. The value of the corresponding radius $R_{\mathrm{c}}=6 \mathrm{~km}$ is slightly smaller than the value $\sim 9.6 \mathrm{~km}$ usually reported (see, e.g., Weinberg 1972) and the difference in attributed in part to the presence of the envelope which surrounds the isothermal region of size $R_{\mathrm{c}}$. According to Eq. (138), the fractional redshift of a spectral line emitted from the edge of the isothermal region (for the configuration of maximum mass) is

$z^{\mathrm{c}}(R)=\left(1-\frac{2 G M_{\mathrm{c}}}{R_{\mathrm{c}} c^{2}}\right)^{-1 / 2}-1=0.35$.

and the corresponding central redshift $z_{0}^{\mathrm{c}}=1.4$. Finally, we find that the central density at the mass peak is $\epsilon_{0}^{\mathrm{c}}=10^{16} \mathrm{gc}^{2} / \mathrm{cm}^{3}$ is good agreement with the reference model of Meltzer \& Thorne (1966) giving a comparable value. Other central densitites are indicated in Fig. 15. Our curve matches relatively well (qualitatively and semiquantitatively) the diagram of Meltzer \& Thorne (1966) for central densities $\epsilon_{0}>4 \times 10^{15} \mathrm{gc}^{2} / \mathrm{cm}^{3}$.

\section{Dynamical instability of isothermal gas spheres in general relativity}

\subsection{The condition of marginal stability}

The stability of relativistic stars against radial perturbations is usually studied with the equation of pulsations derived by Chandrasekhar (1964). Then, the stability of stars can be reduced to a Sturm-Liouville problem, which is usually solved numerically (see, e.g., Bardeen et al. 1966). The method is quite general but it is not the most efficient in the present situation. Indeed, by formulating the problem differently, using the form of the equation of pulsations given by Yabushita (1973) and making use of the Milne variables, it is possible to study the stability of bounded isothermal spheres analytically, like in the Newtonian case.

Let us consider a small perturbation $\delta \epsilon$ around a configuration of isothermal equilibrium and let us introduce the mass perturbation $f(\xi)$ within the sphere of radius $\xi$ such that

$\frac{\delta \epsilon}{\epsilon_{0}}=\frac{1}{4 \pi \xi^{2}} \frac{\mathrm{d} f}{\mathrm{~d} \xi}$.

Yabushita $(1973,1974)$ has shown that the equation for radial pulsations satisfied by the function $f$ can be written

$$
\begin{aligned}
& \frac{\mathrm{d}^{2} f}{\mathrm{~d} \xi^{2}}+\left(-\frac{2}{\xi}+\frac{\mathrm{d} \psi}{\mathrm{d} \xi}+q \xi \mathrm{e}^{\lambda-\psi}\right) \frac{\mathrm{d} f}{\mathrm{~d} \xi} \\
& +\mathrm{e}^{\lambda-\psi}\left[1+\frac{2 q \xi}{1+q} \frac{\mathrm{d} \psi}{\mathrm{d} \xi}\right] f=\frac{\sigma^{2} c^{4}}{4 \pi G \epsilon_{0}(1+q)} \mathrm{e}^{\lambda-\nu} f
\end{aligned}
$$

where $\sigma$ is the period of oscillations defined by $\delta \epsilon \sim \mathrm{e}^{\sigma c t}$. On the other hand, the velocity profile of the perturbation is given by

$\delta u^{1}=-\frac{\sigma c}{q+1} \frac{1}{4 \pi \epsilon} \frac{f}{r^{2}} \mathrm{e}^{-\nu / 2}$.

If the system is confined within a box, we must require that $\delta u^{1}=0$ at $r=R$. The function $f(\xi)$ must therefore satisfy the boundary conditions

$f(0)=f(\alpha)=0$.

These boundary conditions are equivalent to the conservation of the mass-energy $M$. In the Newtonian limit $q \rightarrow 0$, the equation of pulsations (164) reduces to the one considered by Yabushita (1968) and Chavanis (2001). Clearly, the condition of instability corresponds to $\sigma^{2}>0$. We shall be particularly interested by the point of marginal stability $(\sigma=0)$ in the series of equilibrium at which the system becomes unstable. We then have to solve the equation

$$
\begin{aligned}
\frac{\mathrm{d}^{2} F}{\mathrm{~d} \xi^{2}} & +\left(-\frac{2}{\xi}+\frac{\mathrm{d} \psi}{\mathrm{d} \xi}+q \xi \mathrm{e}^{\lambda-\psi}\right) \frac{\mathrm{d} F}{\mathrm{~d} \xi} \\
& +\mathrm{e}^{\lambda-\psi}\left[1+\frac{2 q \xi}{1+q} \frac{\mathrm{d} \psi}{\mathrm{d} \xi}\right] F=0,
\end{aligned}
$$

with $F(0)=F(\alpha)=0$. If we denote by $\mathcal{L}$ the differential operator that appears in Eq. (167), it is possible to show that (Yabushita 1974)

$$
\mathcal{L}(M(\xi))=\mathcal{L}\left(\xi^{3} \mathrm{e}^{-\psi}\right)=\frac{\mathrm{d} \psi}{\mathrm{d} \xi} \frac{\mathrm{d} M}{\mathrm{~d} \xi}+\frac{2 q \xi}{1+q} \mathrm{e}^{\lambda-\psi} M(\xi) \frac{\mathrm{d} \psi}{\mathrm{d} \xi} .
$$

Therefore, the eigenfunction associated with $\sigma=0$ is

$F(\xi)=c_{1}\left(\xi^{3} \mathrm{e}^{-\psi}-M(\xi)\right)$.

In the classical limit, we recover the result $\xi^{3} \mathrm{e}^{-\psi}-\xi^{2} \psi^{\prime}$ derived from the analysis of the second order variations of the free energy (in the canonical ensemble) or from the Navier-Stokes equations (Chavanis 2001). The condition $F(0)=0$ is automatically satisfied. The condition $F(\alpha)=0$ determines the values of $\alpha$ at which a new mode of stability is lost. According to Eq. (169), we get

$M(\alpha)=\alpha^{3} \mathrm{e}^{-\psi(\alpha)}$.

Using Eq. (144) and introducing the Milne variables (139), it is straightforward to check that this relation is equivalent to

$p v_{0}=\frac{1}{u_{0}}-q-1$

which is precisely Eq. (148). Therefore, a new eigenvalue $\sigma^{2}$ becomes positive at the points where $\chi$ is extremum. In particular, the first instability sets in precisely at the point of maximum mass. Isothermal configurations with a central density $\alpha>\alpha_{1}$, a density contrast $\mathcal{R}>\mathcal{R}_{\mathrm{c}}$ or a central redshift $z_{0}>z_{0}^{\mathrm{c}}$ are unstable with respect to radial perturbations. Similar results were obtained by 
Misner \& Zapolsky (1964) who numerically solved the Chandrasekhar equation for radial pulsations applied to their neutron star model. Their method was, however, limited to the fundamental mode and they could not demonstrate that secondary mass peaks correspond to the change of sign for the next eigenvalues (see their discussion). Our method gives an unambiguous definite answer to that question, at least in the framework of our box model. It has to be noted that these results differ from those obtained by Yabushita (1974) who found that instability occurs before the first mass peak (except in the Newtonian case $q=0)$. Now, the three models only differ in the boundary conditions: (i) Yabushita's gas spheres have envelopes which exert a constant pressure. (ii) In our model, the volume of the isothermal region is assumed to be fixed. (iii) Misner \& Zapolsky introduce a more realistic envelope in which the equation of state has a physically admissible form. Our results suggest that imposing a fixed volume (Antonov point of view) is a better approximation than imposing a fixed pressure (Bonnor point of view) since it provides the same condition of stability as the reference model of Misner \& Zapolsky (1964) and other works.

\subsection{Perturbation profiles and secondary instabilities}

The values of $\alpha$ at which a new mode of instability occurs are determined by Eq. (171). For large values of $\alpha$ (high order modes), we can introduce the asymptotic expansion (134) in the Milne variables. Then, after some algebra, we find that the values of $\alpha_{n}$ at which a new instability occurs are asymptotically given by

$$
\begin{aligned}
& \frac{\left(7+42 q-q^{2}\right)^{1 / 2}}{2(1+q)} \ln \alpha_{n}+\delta \\
& \quad=\arctan \left(\frac{q^{2}+20 q+3}{(1+q)\left(7+42 q-q^{2}\right)^{1 / 2}}\right)+n \pi
\end{aligned}
$$

In continuity with the Newtonian limit, they follow the geometric progression

$\alpha_{n} \sim\left[\mathrm{e}^{\frac{2 \pi(1+q)}{\left(7+42 q-q^{2}\right)^{1 / 2}}}\right]^{n} \quad(n \rightarrow+\infty$, integer $)$.

For $q=0,1 / 3,1$ the ratio in the geometric progression is respectively $10.74,6.25$ and 6.13 . It is minimum for $q=7 / 11$ with a value 5.93 .

The profile of the energy density perturbation associated with each instability is obtained by substituting Eq. (169) in Eq. (163). Simplifying the derivative with the aid of Eq. (125) and introducing the Milne variable $v$, we obtain

$\frac{\delta \epsilon}{\epsilon}=\frac{c_{1}}{4 \pi}(2-v)$

The zeros of the profile $\delta \epsilon$ are determined by the intersections between the spiral in the $(u, v)$ plane and the line $v=2$ (see Fig. 11). The first two zeros are represented in Fig. 16 for different values of $q$. We see that $\xi_{2}$ is always larger than $\alpha_{1}$ so it does not satisfy the requirement

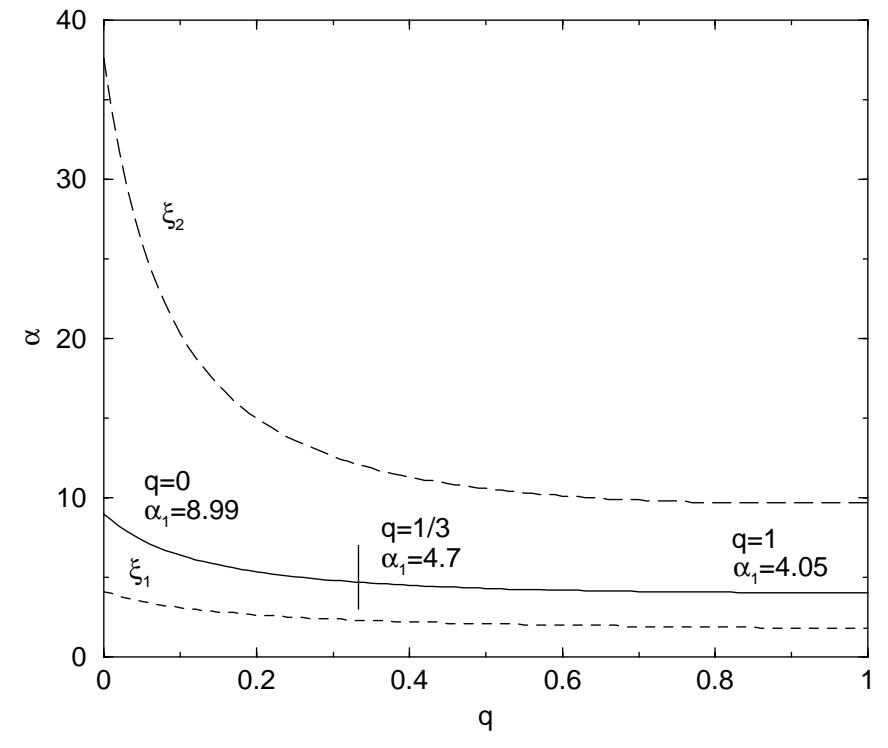

Fig. 16. Critical parameter $\alpha_{1}$ as a function of $q$ (full line) together with the first two zeros of the perturbation profile (174) satisfying $v\left(\xi_{i}\right)=2$ (dashed lines). Since $\xi_{2}>\alpha_{1}$, this solution must be rejected.

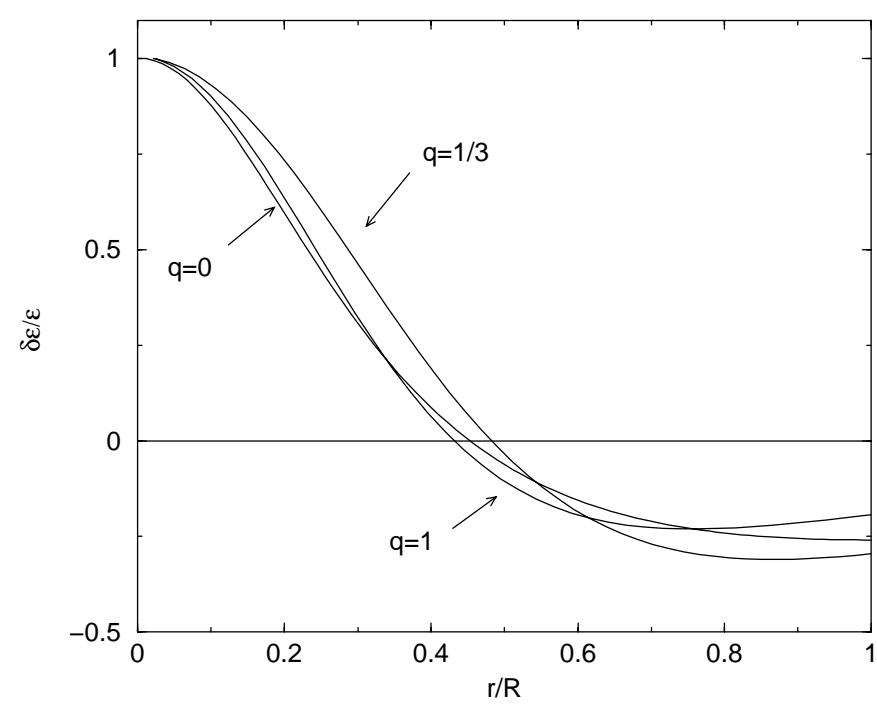

Fig. 17. Density perturbation profiles at the point of marginal stability for different values of $q$. The perturbation profiles do not present a core-halo structure.

$0 \leq \xi \leq \alpha$. Therefore, the first mode of instability has only one node at $\xi=\xi_{1}$. In other words, the perturbation profile that triggers the instability of an isothermal sphere in general relativity does not present a "core-halo" structure (see Fig. 17) in continuity with the Newtonian study in the canonical ensemble (Chavanis 2001).

The high order modes of instability have several nodes. Their positions (for large $\xi_{i}$ ) are obtained by expanding the Milne variable $v$ with the aid of Eq. (134) and substituting the resulting expression in the condition $v=2$. 
This yields

$$
\begin{aligned}
& \frac{\left(7+42 q-q^{2}\right)^{1 / 2}}{2(1+q)} \ln \xi_{i}+\delta \\
& \quad=-\arctan \left(\frac{1+3 q}{\left(7+42 q-q^{2}\right)^{1 / 2}}\right)+i \pi
\end{aligned}
$$

Therefore, the zeros of the density perturbation profiles also follow a geometric progression

$\xi_{i} \sim\left[\mathrm{e}^{\frac{2 \pi(1+q)}{\left(7+42 q-q^{2}\right)^{1 / 2}}}\right]^{i} \quad(i \rightarrow+\infty$, integer $)$,

with the same ratio as the dimensionless radii $\alpha_{n}$.

We can also determine the velocity profile (165) associated with each mode of instability. Introducing the velocity of sound $c_{\mathrm{s}}=q^{1 / 2} c$ and expressing the result in terms of the dimensionless variables defined in Sect. 3.3, we get

$$
\frac{\delta u^{1}}{c_{\mathrm{S}}}=-\frac{c \sigma^{\prime}}{4 \pi(q+1) \xi^{2}} \mathrm{e}^{\frac{\psi(\xi)}{1+q}} F(\xi)
$$

where $F(\xi)$ is given by Eq. (169) at the critical points. In writing Eq. (177), it is implicitly understood that we are just at the onset of instability $\left(\sigma^{\prime}=0^{+}\right)$, so that Eq. (177) is applicable with $\sigma^{\prime}>0$ (the velocity profile at the critical points, i.e. $\sigma^{\prime}=0$, is simply $\delta u^{1}=0$ ). In terms of the Milne variables, the velocity profile (177) can be written

$\frac{\delta u^{1}}{c_{\mathrm{S}}}=-\frac{c \sigma^{\prime}}{4 \pi(q+1)} c_{1} \psi^{\prime} \mathrm{e}^{\frac{\psi(\xi)}{1+q}} \frac{u}{1+p v}\left(p v-\frac{1}{u}+q+1\right)$.

For $q=0$, we recover the expression obtained in the Newtonian context from the Navier-Stokes equations (Chavanis 2001). Since $F(0)=F(\alpha)=0$, the velocity always vanishes at the center and at the boundary of the domain. The other zeros, for which $F\left(\xi_{i}\right)=0$, correspond precisely to the values of $\alpha$ at which a new mode of instability occurs, i.e. $\xi_{i}=\alpha_{i}$. The $n$th mode of instability therefore has $n+1$ zeros $\xi=0, \xi=\alpha_{1}, \xi=\alpha_{2}, \ldots$, $\xi=\alpha_{n}$. For high order modes of instability, the zeros follow asymptotically the geometric progression (173).

\subsection{The baryon number: Another interpretation of the instability}

It has often been suggested on the basis of phenomenological arguments that the baryon number $N$, or equivalently the rest mass $M_{0}=N m$, is also maximum at the onset of instability. We wish to check this prediction explicitly in the case of an isothermal gas sphere.

The baryon number is obtained by multiplying the baryon number density $n$ by the proper volume element $\mathrm{e}^{\lambda(r) / 2} 4 \pi r^{2} \mathrm{~d} r$ and integrating the resulting expression over the whole configuration. Using Eq. (106), we get (see, e.g., Weinberg 1972)

$N=\int_{0}^{R} n\left[1-\frac{2 G M(r)}{r c^{2}}\right]^{-1 / 2} 4 \pi r^{2} \mathrm{~d} r$.

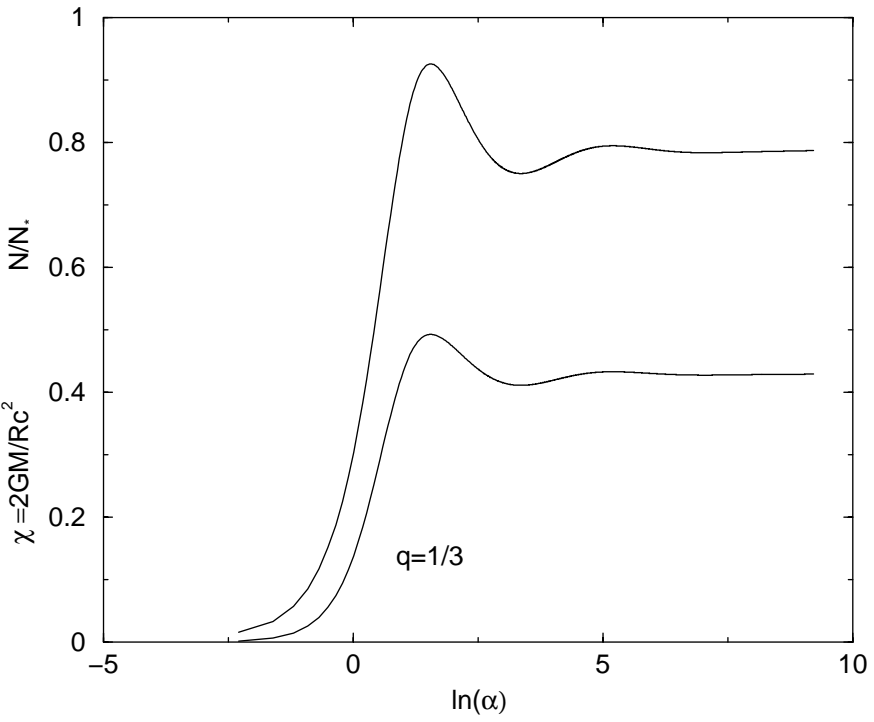

Fig. 18. Baryon number and total mass-energy as a function of the central density (through the parameter $\alpha$ ) for $q=1 / 3$. The peaks occur for the same values of the central density.

Now, according to the equations of state (116)-(118), the baryon number density is related to the energy density by

$n^{\gamma}=\frac{q}{K} \epsilon$

Introducing the dimensionless variables defined in Sect. 3.3, we find that the baryon number can be written

$\frac{N}{N_{*}}=\frac{1}{\alpha^{\frac{3 q+1}{1+q}}} \int_{0}^{\alpha} \mathrm{e}^{-\frac{\psi(\xi)}{1+q}}\left[1-p \frac{M(\xi)}{\xi}\right]^{-1 / 2} \xi^{2} \mathrm{~d} \xi$,

with the normalization constant

$N_{*}=4 \pi R^{3}\left[\frac{q^{2} c^{4}}{4 \pi G K R^{2}(1+q)}\right]^{1 / \gamma}$.

The baryon number (181) is ploted versus the central density in Fig. 18 for $q=1 / 3$. It exhibits damped oscillations, like the total mass energy, and tends asymptotically to the value $\frac{1+q}{3 q+1} Q^{\frac{1}{1+q}}(1-p Q)^{-1 / 2}$ as $\alpha \rightarrow+\infty$ (singular sphere). It is observed numerically that the peaks of mass-energy and of baryon number occur for the same values of the central density (we have checked this property for various values of $q$ ). This is also clear from Fig. 19 where the mass-energy $M$ is ploted as a function of the baryon number $N$. From this figure, we can understand physically why the system becomes unstable after the first mass peak. Indeed, for a given rest mass $N m c^{2}$ there exists several solutions with different energy $M c^{2}$. Only the solution with the lowest energy ("fundamental" state) is stable with respect to radial perturbations (see Weinberg 1972). We expect therefore some phase transitions to occur from a state of high energy to a state of lower energy (see the arrow in Fig. 19). Therefore, only the lower branch is stable and it corresponds to the configurations prior to the first mass peak (we come to the same conclusion by 


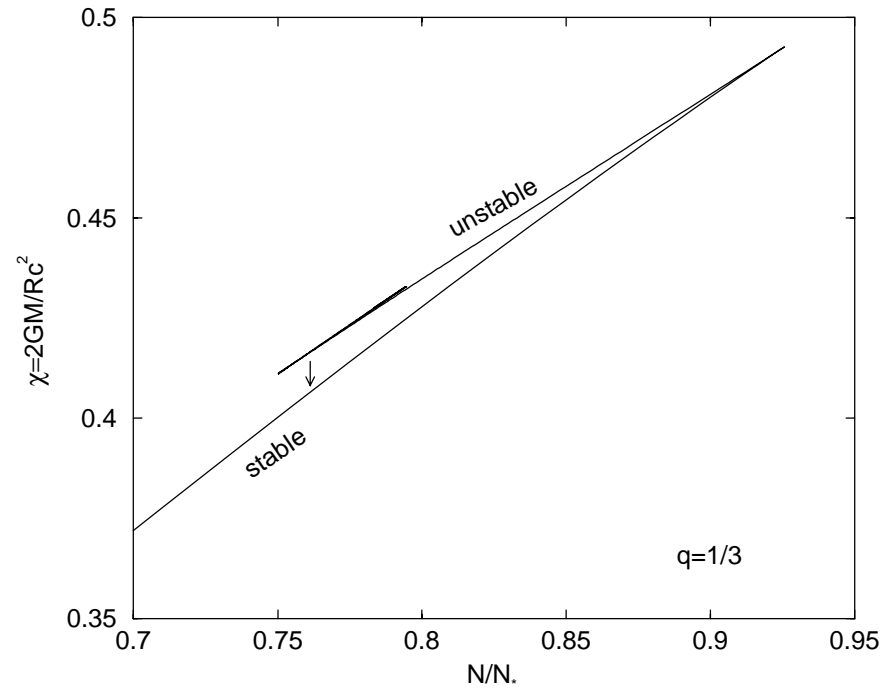

Fig. 19. Total mass-energy versus baryon number for isothermal gaseous spheres $(q=1 / 3)$.

minimizing the binding energy $E=\left(M-M_{0}\right) c^{2}$ at fixed mass $M)$. These results are consistent with those found by Tooper (1964) for polytropic gas spheres.

\subsection{Characteristic length scales}

We shall now reformulate the previous results in a different form by introducing appropriate length scales. This will provide a direct comparison with our discussion in the Newtonian context (Chavanis 2001). In general relativity, a gaseous sphere of mass $M$ is unstable if its radius is smaller than a multiple of the Schwarzschild radius (Chandrasekhar 1964). For a confined isothermal gas, this condition is explicitly given by Eq. (150) which can be rewritten

$R \leq \frac{1}{\chi_{\mathrm{c}}(q)} R_{\mathrm{S}}, \quad R_{\mathrm{S}}=\frac{2 G M}{c^{2}}$.

On the other hand, in Newtonian gravity, the condition of gravitational collapse is that the size of the domain be larger than the Jeans length $L_{\mathrm{J}}$. For example, in the case of an isothermal gas confined within a box, the condition of instability reads (de Vega \& Sanchez 2001; Chavanis 2001)

$R \geq \sqrt{\frac{2.52 \ldots}{3}} L_{\mathrm{J}}, \quad L_{\mathrm{J}}=\left(\frac{3 R^{3}}{\beta G M m}\right)^{1 / 2}$.

It should be emphasized that these inequalities characterize the absence of hydrostatic equilibrium for an isothermal gas beyond these thresholds. This is more stringent than the instability of an equilibrium configuration (see below). Although the sense of these inequalities is opposite, there is no contradiction since the size of the domain enters explicitly in the definition of the Jeans length (through the average density). For $q \rightarrow 0$, the two criteria become equivalent as we now show. In the classical limit, the rest mass dominates the kinetic energy so that $\epsilon=\rho c^{2}$ where $\rho=n m$ is the classical mass density. Therefore, the equation of state (118) becomes $p=q \rho c^{2}$ and it must be compared with the equation of state of a classical isothermal gas $p=\frac{\rho}{m} k T$. This yields

$q=\frac{k T}{m c^{2}}=\frac{1}{\beta m c^{2}} \quad\left(\beta m c^{2} \rightarrow+\infty\right)$.

Now, in the classical limit $c \rightarrow+\infty$, the critical parameter $1 / \chi_{\mathrm{c}}$, given by Eq. (149), diverges like $1 / \chi_{\mathrm{c}}=$ $1 / 2 q v\left(\alpha_{1}\right) \rightarrow+\infty$ while the Schwarzschild radius $R_{\mathrm{S}}$ goes to zero in such a way that the product $\left(1 / \chi_{\mathrm{c}}\right) R_{\mathrm{S}}$ remains finite. Therefore, the inequality (183) is reversed

$R \leq \frac{\beta G M m}{v\left(\alpha_{1}\right)}=\frac{3 R^{3}}{v\left(\alpha_{1}\right) L_{\mathrm{J}}^{2}} \Longrightarrow R \geq \sqrt{\frac{v\left(\alpha_{1}\right)}{3}} L_{\mathrm{J}}$,

and we recover the result $(184)$ with $v\left(\alpha_{1}\right)=2.52$. It can be noted that the same criterion can be written

$M \geq M_{\mathrm{c}} \equiv 2.52 \frac{R k T}{G m}$.

Therefore, a classical gaseous sphere of radius $R$ and temperature $T$ can exist only below a limiting mass $M_{\mathrm{c}}$ given by formula (187). Obviously, the physical origin of this limiting mass is the same as for the limiting mass of neutron stars but with a completely different interpretation of the parameters.

It is also relevant to introduce another length scale

$L_{\mathrm{c}}=\left(\frac{9 q c^{4}}{4 \pi G \epsilon_{0}(1+q)}\right)^{1 / 2}$

which gives a good estimate of the "core radius" of a relativistic isothermal gas sphere. In the classical limit, $L_{\mathrm{c}}$ reduces to the King's length (Binney \& Tremaine 1987). In terms of the core radius, the parameter $\alpha$ can be written

$\alpha=3 \frac{R}{L_{\mathrm{c}}}$.

The condition of instability $\alpha>\alpha_{1}$ is therefore equivalent to

$R>\frac{\alpha_{1}}{3} L_{\mathrm{c}}$.

Above this threshold, it is still possible to construct isothermal gas spheres in hydrostatic equilibrium but they are unstable. Secondary instabilities occur for radii that follow a geometric progression

$R_{n} \sim\left[\mathrm{e}^{\frac{2 \pi(1+q)}{\left(7+42 q-q^{2}\right)^{1 / 2}}}\right]^{n} L_{\mathrm{c}} \quad(n \rightarrow+\infty$, integer $)$.

In the Newtonian context, this hierarchy of scales was interpreted by Semelin et al. (1999) and Chavanis (2001) in relation with the fragmentation of the interstellar medium and the possible origin of its fractal structure.

The instability criteria (183) and (190), expressed in terms of distinct length scales, can also be expressed in terms of the redshift, with a similar distinction between 
the central redshift and the surface redshift. Indeed, the surface redshift $z(R)$ is an increasing function of the mass energy $\chi$ while the central redshift $z_{0}$ is an increasing function of the central density $\alpha$. Therefore, the surface redshift must necessarily satisfy $z(R)<z(R)^{\mathrm{c}}$ since no hydrostatic equilibrium of isothermal spheres can exist above this threshold while the central redshift must satisfy $z_{0}<z_{0}^{\mathrm{c}}$ because configurations with $z_{0}>z_{0}^{\mathrm{c}}$ exist mathematically but are unstable.

\section{Conclusion}

In this paper, we have studied the structure and the stability of isothermal gas spheres in the framework of general relativity. We have found that relativistic isothermal spheres (like neutron cores) exhibit the same kind of behaviors as Newtonian isothermal spheres but with a different interpretation of the parameters. In this analogy, the critical energy and the critical temperature found by Antonov (1962) and Lynden-Bell \& Wood (1968) for an isothermal gas are in some sense the classical equivalent of the limiting mass of neutron stars discovered by Oppenheimer \& Volkoff (1939) (there also exists a limiting mass for classical isothermal gas spheres with given temperature and radius, see Eq. (187)). In addition, the spiral behavior of the temperature-energy curve for a classical gas has the same origin as the mass-radius diagram for neutron stars. This spiral behavior, as well as the damped oscillations of the mass-density profile, are general features of isothermal configurations.

Although our model can reproduce qualitatively the main properties of neutron stars, it is however limited in its applications since the isothermal core is surrounded by an artificial "box" instead of a more physical envelope like in the studies of Oppenheimer \& Volkoff (1939), Misner \& Zapolsky (1964) and Meltzer \& Thorne (1966). However, this simplification allows us to study the stability problem analytically (or with graphical constructions) without any further approximation. This is a useful complement to the more elaborate works of the previous authors who had to solve the pulsation equation numerically or with approximations. This is also complementary to the study of Yabushita (1974) who considered isothermal gas spheres surrounded by an envelope exerting a constant pressure. The boundary conditions are of importance since Yabushita came to the conclusion that the onset of instability occurs before the first mass peak. In contrast, our model gives a stability criterion consistent with that of Misner \& Zapolsky (1964) and other works. Furthermore, if we assume that the energy density at the edge of the isothermal core has a value $\sim 10^{15} \mathrm{gc}^{2} / \mathrm{cm}^{3}$ (a typical prediction of nuclear models), we can reproduce almost quantitatively the results usually reported for neutron stars.

It should be stressed that the analogy between neutron stars and isothermal spheres is a pure effect of general relativity. When gravity is treated in the Newtonian framework, the classical condition of hydrostatic equilibrium requires a relationship between the pressure and the mass density $\rho=m n$ which, for dense matter (degenerate and relativistic), is a power law with index $\gamma=4 / 3$ (see Chandrasekhar 1942). However, when gravity is treated in the framework of general relativity, the OppenheimerVolkoff equations require a relationship between the pressure and the mass-energy density $\epsilon$ which, for dense matter, is linear. Therefore, the core of neutron stars (treated with general relativity) is "isothermal" while the core of white dwarf stars approaching the limiting mass (treated in a Newtonian framework) is "polytropic", although the same equation of state is used (that for a completely degenerate and ultra-relativistic ideal Fermi gas). This is the intrinsic reason why the Mass-Radius relation for neutron stars exhibits a spiral behavior (Meltzner \& Thorne 1966) while the Mass-Radius relation for white dwarf stars is monotonous (Chandrasekhar 1942). These remarks may increase the interest of studying isothermal gas spheres both in Newtonian mechanics and in general relativity.

Acknowledgements. This work was initiated during my stay at the Institute for Theoretical Physics, Santa Barbara, during the program on Hydrodynamical and Astrophysical Turbulence (February-June 2000). This research was supported in part by the National Science Foundation under Grant No. PHY94-07194.

\section{References}

Ambartsumyan, V. A., \& Saakyan, G. S. 1961, Sov. Astron. AJ, 4, 187

Antonov, V. A. 1962, Vest. Leningr. Gos. Univ., 7, 135

Bardeen, J. M., Thorne, K. S., \& Meltzer, D. W. 1966, ApJ, 145,505

Binney, J., \& Tremaine, S. 1987, Galactic Dynamics (Princeton Series in Astrophysics)

Bisnovatyi-Kogan, G. S., Merafina, M., Ruffini, R., \& Vesperini, E. 1998, ApJ, 500, 217

Bisnovatyi-Kogan, G. S., \& Thorne, K. S. 1970, ApJ, 160, 875

Bisnovatyi-Kogan, G. S., \& Zel'dovich, Ya. B. 1969, Astrofizika, 5, 223

Bonnor, G. S. 1956, MNRAS, 116, 351

Buchdahl, H. A. 1959, Phys. Rev., 116, 1027

Chandrasekhar, S. 1942, An Introduction to the Theory of Stellar Structure (Dover)

Chandrasekhar, S. 1964, ApJ, 140, 417

Chandrasekhar, S. 1972, A limiting case of relativistic equilibrium in General Relativity, papers in honour of J. L. Synge, ed. L. O' Raifeartaigh (Oxford)

Chavanis, P. H. 2001, to appear in A\&A [astro-ph/0103159]

Chavanis, P. H., Rosier, S., \& Sire, C. 2001, submitted to Phys. Rev. E [cond-mat/0107345]

de Vega, H. J., Sanchez, N., \& Combes, F. 1996, Phys. Rev. $\mathrm{D}, 54,6008$

de Vega, H. J., Sanchez, N., \& Combes, F. 1998, ApJ, 500, 8 de Vega, H. J., \& Sanchez, N. 2001 [astro-ph/0101568]

Hjorth, J., \& Madsen, J. 1993, MNRAS, 265, 237

Katz, J. 1978, MNRAS, 183, 765

Katz, J. 1980, MNRAS, 190, 497

Katz, J., Horwitz, G., \& Klapisch, M. 1975, ApJ, 199, 307 
Lynden-Bell, D. 1967, MNRAS, 136, 101

Lynden-Bell, D., \& Wood, R. 1968, MNRAS, 138, 495

Meltzer, D. W., \& Thorne, K. S. 1966, ApJ, 145, 514

Misner, C. W., Thorne, K. S., \& Wheeler, J. A. 1973, Gravitation (Freeman)

Misner, C. W., \& Zapolsky, H. S. 1964, Phys. Rev. Lett., 12, 635

Oppenheimer, J. R., \& Volkoff, G. M. 1939, Phys. Rev., 55, 374

Padmanabhan, T. 1989, ApJS, 71, 651

Padmanabhan, T. 1990, Phys. Rep., 188, 285

Pfenniger, D., \& Combes, F. 1994, A\&A, 285, 94

Saslaw, W. C., \& Hamilton, A. J. S. 1984, ApJ, 276, 13

Saslaw, W. C., Maharaj, S. D., \& Dadhich, N. 1996, ApJ, 471, 571
Semelin, B., de Vega, H. J., Sanchez, N., \& Combes, F. 1999, Phys. Rev. D, 59, 125021

Semelin, B., Sanchez, N., \& de Vega, H. J. 2001, Phys. Rev. $\mathrm{D}, 63,084005$

Tolman, R. 1934, Relativity, Thermodynamics and Cosmology (Oxford: Clarendon Press)

Tooper, R. 1964, ApJ, 140, 434

Tooper, R. 1965, ApJ, 142, 1541

Tsuruta, S., \& Cameron, A. G. W. 1966, Can. J. Phys., 44, 1895

Weinberg, S. 1972, Gravitation and cosmology (John Wiley)

Yabushita, S. 1968, MNRAS, 140, 109

Yabushita, S. 1973, MNRAS, 165, 17

Yabushita, S. 1974, MNRAS, 167, 95

Zel'dovich, Ya. B. 1962, Sov. Phys. JETP, 14, 1143 